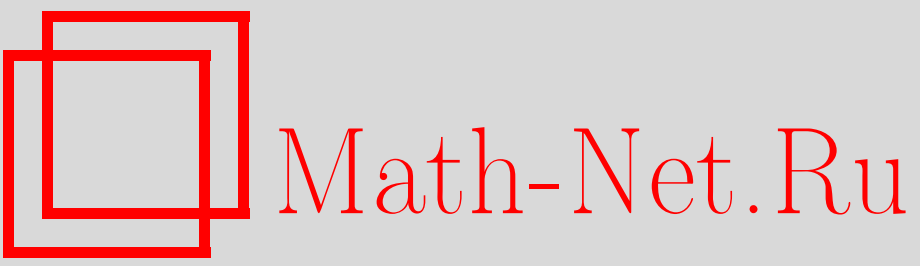

В. Н. Соловьев, Двойственные экстремальные задачи и их применения к задачам минимаксного оценивания, УМН, 1997, том 52, выпуск 4, 49-86

DOI: https://doi.org/10.4213/rm863

Использование Общероссийского математического портала Math-Net.Ru подразумевает, что вы прочитали и согласны с пользовательским соглашением

http://www . mathnet.ru/rus/agreement

Параметры загрузки:

IP: 3.80 .181 .102

26 апреля 2023 г., 14:44:58 


\title{
ДВОЙСТВЕННЫЕ ЭКСТРЕМАЛЬНЫЕ ЗАДАЧИ И ИХ ПРИМЕНЕНИЯ К ЗАДАЧАМ МИНИМАКСНОГО ОЦЕНИВАНИЯ
}

\author{
В. Н. Соловьёв
}

\section{СОДЕРЖАНИЕ}

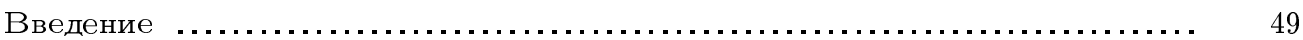

Глава 1. Двойственность невыпуклых экстремальных задач ............ 51

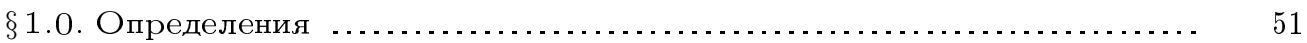

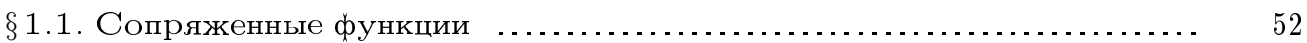

$\S 1.2$. Характеризация выпуклости в терминах гладкости $\ldots . \ldots \ldots \ldots \ldots . . . . . . .56$

$\S 1.3$. Теоремы о минимаксе .................................... 60

$\S 1.4$. Сопряженная функция к максимуму семейства квадратичных форм 62

$\S 1.5$. Двойственность невыпуклых экстремальных задач в случае глад-

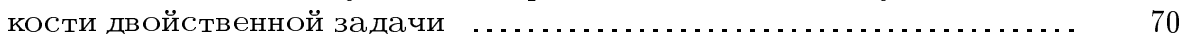

Глава 2. Минимаксное оценивание параметров в линейных моделях наблюдения с неопределенными статистиками второго порядка .... 71

$\S 2.1$. Постановка задачи ..................................... 71

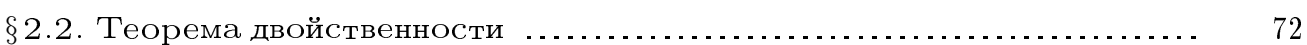

$\S 2.3$. Минимаксные оценки и метод наименьших квадратов . ............. 73

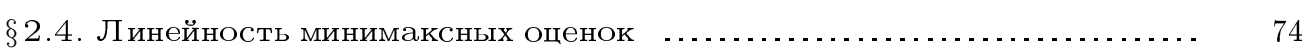

Глава 3. Приложения к теории плохо обусловленных задач $\ldots . . . \ldots . . . .675$

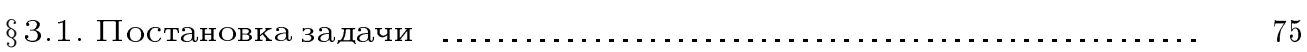

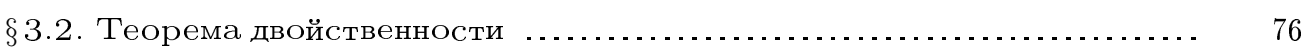

$\S 3.3$. Оценка Кукса-Ольмана ............................... 77

$\S 3.4$. Несимметричные ограничения на параметры регрессии . ........ 81

Список литературы ............................................... 83

\section{Введение}

Основы теории двойственности в выпуклом анализе заложены еще в классических работах Г. Минковского, Г. Хана, Дж. фон Неймана, Э. Хелли. Эта теория приобрела стройные очертания после фундаментальных работ В. Фенхеля и Р.Т. Рокафеллара, в которых были введены и систематически изучены свойства сопряженных 
функций. Вьпукльй анализ занял видное место в современной теории экстремальных задач (см. монографии В.М. Алексеева, В.М. Тихомирова и С.В. Фомина [2], А. Д. Иоффе и В. М. Тихомирова [19], Е. Г. Гольштейна [96], Ж.-П. Обена и И. Экланда [39], Б. Т. Поляка [42], Б.Н. Пшеничного [43], Р. Т. Рокафеллара [45], И. Экланда и Р. Темама [65]).

Настоящая работа посвящена тому фрагменту теории двойственности, в котором важную роль играет связь выпуклости в исходном пространстве с гладкостью в сопряженном пространстве. По-видимому, впервые связь двойственности с гладкостью была установлена В. Л. Шмульяном [62], которьй обнаружил, что банахово пространство рефлексивно, если норма в сопряженном пространстве дифференцируема по Фреше. Попутно обнаружилось, что в этом случае единичньй шар исходного пространства является равномерно (или строго) вьпуклым (граница этого шара не содержит прямолинейных отрезков; аналитическую формулировку см., например, в [97, с. 510]). Связь строгой вьпуклости в исходном пространстве с гладкостью в сопряженном пространстве затем исследовалась М. Дэем, В. Кли, Е. Асплундом, Р. Т. Рокафелларом и другими. Много позднее и в других терминах связь двойственности с гладкостью была также отмечена в задачах минимаксного оценивания (С. Верду и Г.В. Пур [84], И.Ф. Пинелис [40], [41]). Наше изложение основано на работах [47], [87], [88], возникших в связи с различными приложениями теории двойственности экстремальных задач (в частности, к задачам минимаксного оценивания), в которых систематически использовался аппарат сопряженных функций.

Основная идея работы [47] состояла в том, что гладкость в сопряженном пространстве автоматически влечет за собой выпуклость в исходном пространстве, даже если об этом заранее ничего не было известно. Однако затем обнаружилось, что такого рода результаты совсем просто следуют из фундаментальных результатов Е. Асплунда и Р.Т. Рокафеллара [66], [67] о связи строгой выпуклости (rotundity) в исходном пространстве с гладкостью в сопряженном (см. теоремы 1.1.0 и 1.1.1 ниже).

В $\S 1.1$ показано, что для полунепрерьвных снизу функций, даже без вьпуклости, в некоторых точках $y_{0}$ должна сохраняться двойственность (т.е. значения функции в этих точках совпадают со значениями второй сопряженной функщии). С помошью этого факта в $§ 1.2$ показано, что произвольная (слабо) полунепрерьвная снизу функция, заданная на рефлексивном банаховом пространстве, будет выпуклой, если ее сопряженная дифференцируема по (Гато) Фреше. В свою очередь, этот результат позволяет доказать средствами вьпуклого анализа тонкие теоремы о выпуклости чебьшёвских множеств, принадлежащие Н. В. Ефимову и С. Б. Стечкину [15]. В 1.3 обсуждаются теоремы о минимаксности без условий вогнутости, впервые обнаруженные С. Верду [47]. Эти результаты являются аналогами теорем из $§ 1.1$. Обширный $§ 1.4$ посвящен вычислению сопряженных функций к максимуму семейств квадратичных форм. Это имеет многочисленные применения в анализе и статистике [87], [88]. В 1.5 результаты $\S 1.1$ применяются к теории двойственных экстремальных задач [87]. Здесь основной результат состоит в том, что разрьвы двойственности должны быть связаны с негладкостью двойственной задачи (двойственная задача либо вообще не имеет решения, либо она не дифференцируема в точке своего минимума).

Глава 2 посвящена задаче минимаксного оценивания параметров в линейных регрессионных моделях с неопределенными статистиками второго порядка. Различные 
постановки задач минимаксного оценивания были независимо предложены почти в одно и то же время (около 1963 года) А.Н. Колмогоровьм [30], Н.Н. Красовским [22], М. Л. Лидовым [32] и П. Хьюбером [60]. Эти задачи интересны не только тем, что здесь была обнаружена связь двойственности с гладкостью, но прежде всего тем, что они дают одно из самых ярких свидетельств в пользу применения теории двойственности. В $\S 2.2$ вместо исходной негладкой задачи, в которой может быть очень много переменных, осуществляется переход к двойственной задаче, в которой сопряженная функция непрерьвно дифференцируема и для ряда практически важных случаев статистических ограничений может быть вычислена аналитически. В этих случаях двойственная задача заключается в безусловной минимизации эффективно вычисляемой гладкой выпуклой функции вектора состояния, которая имеет небольшую размерность [8], [48], [50], [52]. В $§ 2.3$ показано, что решением минимаксной задачи является оценка наименьших квадратов, соответствуюшая наименее благоприятной матрище вторых моментов (результат, по-видимому, восходящий еше к Вальду [90]). С его помошью в конце главы 2 установлена линейность минимаксных оценок скалярного параметра.

В главе 3 рассматривается задача восстановления параметра $l=\langle c, \theta\rangle$ из вектора данных $\eta=A \theta+\xi$ при наличии априорной информации $\theta \in \Theta$. Из обширной теории плохо обусловленных задач наиболее близкими по постановкам и подходам работами являются [29], [58]. Сначала мы сосредоточимся на случае центрально-симметричного множества $\Theta$. В $\S 3.2$ дано конечномерное обобщение одного важного результата, принадлежащего И. А. Ибрагимову и Р.З. Хасьминскому [17], [18], а также А. М. Федотову [58], на задачи минимаксного оценивания с неопределенными статистиками второго порядка. В $\S 3.3$ приведено обобщение красивого результата, принадлежашего В.Б. Меласу [38], которое в случае точно определенных статистик первого и второго порядка приводит к аномальной двойственной задаче. В конще главы 3 показано, что если априорное множество $\Theta$ не симметрично относительно нуля, все результаты этой главы по-прежнему применимы, но множество $\Theta$ следует симметризовать относительно нуля. В доказательствах используются теоремы о минимаксе из $§ 1.3$ (стандартными теоремами о минимаксе в этих задачах воспользоватьсяне удается (см. [17], [18], [58])).

Поскольку одной из целей этой работы было продемонстрировать, как в разных ситуациях работают одни и те же методы, и так как многие результаты в ней публикуются впервые, то обычно (исключая главу 2), они даны с доказательствами. Автор осознает, что неизбежно чьи-то труды не были отражены в этом обзоре, и заранее приносит их авторам свои искренние извинения.

\section{Глава 1.}

\section{Двойственность невыпуклых экстремальных задач}

\section{§1.0. Определения}

Пусть $Y$ - локально выпуклое линейное топологическое (или нормированное) пространство, $Y^{*}$ - его топологически сопряженное и $\left\langle y, y^{*}\right\rangle$ - значение функционала $y^{*} \in Y^{*}$ на элементе $y \in Y$. Как известно, билинейная форма $\left\langle y, y^{*}\right\rangle$ осуществляет двойственность между $Y$ и $Y^{*}$. Для произвольной функции $g(y): Y \rightarrow \overline{\mathbb{R}}:=$ 
$\mathbb{R}^{1} \cup\{+\infty\}$ определим сопряженную и вторую сопряженную (бисопряженную) функции соотношениями

$$
g^{*}\left(y^{*}\right):=\sup _{y \in Y}\left\{\left\langle y, y^{*}\right\rangle-g(y)\right\}
$$

и

$$
g^{* *}(y):=\sup _{y^{*} \in Y^{*}}\left\{\left\langle y, y^{*}\right\rangle-g^{*}\left(y^{*}\right)\right\}
$$

соответственно. Легко видеть, что всегда

$$
g(y) \geqslant g^{* *}(y), \quad g^{*}\left(y^{*}\right)+g^{* *}(y) \geqslant\left\langle y^{*}, y\right\rangle .
$$

Говорят, что функция $g(y): Y \rightarrow \overline{\mathbb{R}}$ полунепрерывна снизу (далее - пн. снизу) в точке $y_{0} \in Y$ (в которой $g\left(y_{0}\right)<+\infty$ ), если для любого $\varepsilon>0$ существует окрестность $V$ точки $y_{0}$ такая, что $g(y) \geqslant g\left(y_{0}\right)-\varepsilon$ при всех $y \in V$.

Напомним, что для вьпуклой функции $g$ при всех $y \in Y$ существует предел

$$
g^{\prime}\left(y_{0} ; y\right):=\lim _{\lambda \rightarrow+0}\left(g\left(y_{0}+\lambda y\right)-g\left(y_{0}\right)\right) / \lambda
$$

которьй назьвается производной функиии $g$ по направлению $y$. Если $g^{\prime}\left(y_{0} ; y\right)=$ $-g^{\prime}\left(y_{0} ;-y\right)$, то этапроизводная называется двусторонней. Если же $g^{\prime}\left(y_{0} ; y\right)=\left\langle y, y_{0}^{*}\right\rangle$ для некоторого элемента $y_{0}^{*} \in Y^{*}$ при всех $y \in Y$, то функция $g$ назьвается дифференцируемой по Гато в точке $y_{0}$. Если, более того, предел (0.3) сушествует равномерно по всем $y$ из единичной сферы пространства $Y$, то функщия $g$ назьвается дифференцируемой по Фреше в точке $y_{0}$. Элемент $y_{0}^{*} \in Y^{*}$ такой, что $g^{\prime}\left(y_{0} ; y\right)=\left\langle y, y_{0}^{*}\right\rangle$, назьвается производной (Фреше или Гато) функции $g$ в точке $y_{0}$.

Аналогично определяются производные Фреше и Гато для сопряженной функции $g^{*}\left(y^{*}\right)$, с той лишш разницей, что теперь производные принадлежат второму сопряженному пространству $Y^{* *}$. Будем говорить, что элемент $y_{0} \in Y$ является производной Фреше (или Гато) сопряженной функции $g^{*}\left(y^{*}\right)$ в точке $y_{0}^{*} \in Y^{*}$, если $g^{*}$ дифференщируема по Фреше (или Гато), причем $g^{* \prime}\left(y_{0}^{*} ; y^{*}\right)=\left\langle y_{0}, y^{*}\right\rangle$ при всех $y^{*} \in Y^{*}($ см. также замечание после теоремы 1.1.1).

\section{$\S$ 1.1. Сопряженные функции}

В вьпуклом анализе и его приложениях основополагающую роль играет равенство

$$
g^{* *}\left(y_{0}\right)=g\left(y_{0}\right)
$$

справедливое для вьпуклой полунепрерьвной снизу функции $g(y)$ во всех точках $y_{0} \in Y$ (см. [2], [19], [45], [65]). Отправной точкой этой работы является следующий фундаментальный результат Е. Асплунда и Р. Т. Рокафеллара [66], [67].

ТЕОРема 1.1.0. Элемент $y_{0} \in Y$ тогда и только тогда является производной Фреше (Гато) сопряженной функиии $g^{*}$ в точке $y_{0}^{*} \in Y^{*}$, когда функция у $\rightarrow$ $\left\langle y_{0}^{*}, y\right\rangle-g^{* *}(y)$ достигает максимума в точке $y_{0}$ и любая ее максимизирующая последовательность сходится (слабо сходится) $к y_{0}$. 
ЗАМЕчАнИЕ. В этой теореме и далее параллельно даются два утверждения: одно из них для случая дифференцируемости по Фреше и пн. снизу в нормированной топологии, а другое для случая дифференцируемости по Гато и пн. снизу в слабой топологии.

Отсюда сразу следует, что даже при отсутствии вьпуклости равенство (1.1) остается справедливым в некоторых точках $y_{0}$.

Tеорема 1.1.1. Пусть произвольная функиия $g(y): Y \rightarrow \overline{\mathbb{R}}$ пн. снизу (слабо пн. снизу) в точке $y_{0} \in Y$, и при этом точка $y_{0}$ является производной Фреше (Гато) сопряженной функции $g^{*}$ в некоторой точке $y_{0}^{*} \in Y^{*}$. Тогда справедливо равенство (1.1) [47], [87].

ДокАЗАТЕЛЬСТвО. Пусть $\left\{y_{k}\right\}$ - максимизирующая последовательность для (0.1) в точке $y^{*}=y_{0}^{*}$. Тогда в силу $(0.2)$ получаем, что

$$
0 \leqslant g^{*}\left(y_{0}^{*}\right)+g^{* *}\left(y_{k}\right)-\left\langle y_{0}^{*}, y_{k}\right\rangle \leqslant g^{*}\left(y_{0}^{*}\right)+g\left(y_{k}\right)-\left\langle y_{0}^{*}, y_{k}\right\rangle \rightarrow 0
$$

при $k \rightarrow \infty$, поэтому

$$
0 \leqslant g^{*}\left(y_{0}^{*}\right)+g^{* *}\left(y_{k}\right)-\left\langle y_{0}^{*}, y_{k}\right\rangle \rightarrow 0 \text { при } k \rightarrow \infty .
$$

Это означает, что $\left\{y_{k}\right\}$ - максимизирующая последовательность функции $y \rightarrow\left\langle y_{0}^{*}, y\right\rangle-$ $g^{* *}(y)$. По теореме 1.1 .0 она сходится (слабо сходится) к $y_{0}$. Так как $g(y)$ пн. снизу (слабо пн. снизу) в точке $y_{0}$, из предыдущего неравенства получаем

$$
g^{*}\left(y_{0}^{*}\right)+g\left(y_{0}\right)-\left\langle y_{0}^{*}, y_{0}\right\rangle \leqslant 0 .
$$

Если бы равенство (1.1) было не верно, мы бы имели

$$
g^{*}\left(y_{0}^{*}\right)+g^{* *}\left(y_{0}\right)-\left\langle y_{0}^{*}, y_{0}\right\rangle<0,
$$

что противоречит (0.2).

ЗАмЕчАнИя. 1) Полунепрерьвность сопряженной функции $g^{*}$ можно понимать в смысле последовательностей. 2) Производные Фреше (Гато) сопряженной функции $g^{*}$ заведомо принадлежат исходному пространству $Y$, а не просто $Y^{* *}$, если $Y$ полно (слабо секвенциально полно) (см. [67, с. 451]).

СледСтвИЕ 1.1.2. Если функиия $g(y): Y \rightarrow \overline{\mathbb{R}}$ пн. снизу (слабо пн. снизу) в нуле и сопряжснная функиия Фреше (Гато) дифференцируема в точке своего минимума, то [87]

$$
g^{* *}(0)=g(0) .
$$

ДокАЗАТЕльство. Предполагается, что точка минимума $y_{0}^{*}$ сопряженной функции $g^{*}\left(y^{*}\right)$ сушествует. Поскольку $0 \in \partial g^{*}\left(y_{0}^{*}\right)$, отсюда и следует результат. 
СлЕДСТВИЕ 1.1.3. Если функиия $g(y): \mathbb{R}^{n} \rightarrow \overline{\mathbb{R}}$ пн. снизу, то двойственность (1.1) вьполняется для всех крайних точек $y_{0}$ субдифференциалов $\partial g^{*}\left(y^{*}\right)$, $y^{*} \in \mathbb{R}^{n}[87]$.

Следующий пример показывает, что в теореме 1.1.1 нельзя заменить производную по Фреше на производную по Гато, если полунепрерьвность снизу имеет место лишш в нормированной, но не слабой топологии.

ПРИмеР БольЦА. Рассмотрим гильбертово пространство $Y=H_{0}^{1}(0,1)$ абсолютно непрерывных функций $y(t):[0,1] \rightarrow \mathbb{R}$, равных нулю на концах отрезка $[0,1]$ и имеющих производную $\dot{y}(t) \in L_{2}(0,1)$. Как известно (см. $[2$, с. 71$]$ и $[39$, с. 24]), функционал

$$
g(y):=\int_{0}^{1}\left\{y^{2}(t)+\left[1-(\dot{y}(t))^{2}\right]^{2}\right\} d t: Y \rightarrow \overline{\mathbb{R}}
$$

пн. снизу в нормированной топологии, но задача

$$
\inf _{y \in Y} g(y)
$$

минимизации $g$ не имеет решения. Мы покажем, что релаксированная задача минимизации бисопряженного функционала $g^{* *}$ имеет единственное решение $y_{0}(t) \equiv 0$. Поскольку множество всех точек минимума функционала $g^{* *}$ совпадает с субдифференциалом $\partial g^{*}(0)$ и, как мы покажем, сопряженньй функционал $g^{*}$ непрерывен, то $y_{0}(t) \equiv 0-$ производная по Гато сопряженного функционала $g^{*}$ в нуле [65]. В то же время $1=g(0)>g^{* *}(0)=0$.

Введем обозначения $u(\alpha):=\left(\alpha^{2}-1\right)^{2}, \alpha_{+}:=\max (\alpha, 0)$,

$$
\tilde{g}(y):=\int_{0}^{1}\left[y^{2}(t)+u^{* *}(\dot{y}(t))\right] d t: Y \rightarrow \overline{\mathbb{R}} .
$$

Так как вторая сопряженная $u^{* *}(\alpha)$, равная $\left[\left(\alpha^{2}-1\right)_{+}\right]^{2}$, неотрицательна, то $g(y) \geqslant$ $\tilde{g}(y) \geqslant 0 \forall y \in Y$. А поскольку вьпуклый функционал $\tilde{g}(y)$ так же, как и $g(y)$, пн. снизу, то

$$
g(y) \geqslant g^{* *}(y) \geqslant \tilde{g}(y) \geqslant 0 \quad \forall y \in Y .
$$

Покажем, что задача минимизации функционала $g^{* *}(y)$ на $Y$ имеет единственное решение $y_{0}(t) \equiv 0$. Для этого рассмотрим минимизируюшую последовательность $\left\{y_{n}(t)\right\}$ в задаче $(1.3)$ :

$$
y_{n}(0)=0, \quad \dot{y}_{n}(t)=(-1)^{k-1}, \quad \frac{k-1}{2 n}<t<\frac{k}{2 n}, \quad k=1,2, \ldots, 2 n, \quad n=1,2, \ldots,
$$

принадлежащую гильбертову пространству $Y=H_{0}^{1}(0,1)$ и равномерно сходящуюся к нулю [19], [39]. Она ограничена в $Y$ :

$$
\left\|y_{n}\right\|^{2}=\int_{0}^{1} \dot{y}_{n}^{2}(t) d t=1 \quad \forall n \in \mathbb{N},
$$


и сходится к нулю на множестве бесконечно дифференцируемых функций $z(t) \in$ $Y^{*}=Y$, которое плотно в $Y^{*}$ :

$$
\left\langle y_{n}, z\right\rangle=\int_{0}^{1} \dot{y}_{n}(t) \dot{z}(t) d t=-\int_{0}^{1} y_{n}(t) \ddot{z}(t) d t \rightarrow 0 \text { при } n \rightarrow \infty,
$$

так как последовательность $y_{n}(\cdot)$ равномерно сходится к нулю и функция $\ddot{z}(t)$ ограничена на отрезке $[0,1]$. Поэтому последовательность $y_{n}(\cdot)$ слабо сходится к нулю (см. [21, с. 195]). Поскольку $g\left(y_{n}\right) \rightarrow 0$ при $n \rightarrow \infty[19]$, [39] и выпукльй пн. снизу функционал $g^{* *}(y)$ пн. снизу в слабой топологии, то с учетом $(1.4)$ имеем

$$
0=\lim _{n \rightarrow \infty} g\left(y_{n}\right) \geqslant \varliminf_{n \rightarrow \infty} g^{* *}\left(y_{n}\right) \geqslant g^{* *}(0) \geqslant 0,
$$

т.е. $g^{* *}(0)=0$. С другой стороны, если $g^{* *}(y)=0$, то в силу (1.4) $\tilde{g}(y)=0$. Но функционал $\tilde{g}(y)$ равен нулю только в точке $y_{0}(t) \equiv 0$, т.е. $y_{0}(t) \equiv 0$ - единственная точка минимума функционала $g^{* *}(y)$ на $Y$.

Таким образом, субдифференциал $\partial g^{*}(0)=\{0\}$ состоит из единственного элемента. Если мы покажем, что сопряженный функционал $g^{*}\left(y^{*}\right)$ непрерьвен, то это будет означать, что $y_{0}(t) \equiv 0$ - производная по Гато функционала $g^{*}\left(y^{*}\right)$ в нуле (см. [65, с. 33]).

Рассмотрим функционал

$$
h(y):=\int_{0}^{1} u(\dot{y}(t)) d t: Y \rightarrow \overline{\mathbb{R}} .
$$

Поскольку $g(y) \geqslant h(y)$ для любого $y \in Y$, то $g^{*}\left(y^{*}\right) \leqslant h^{*}\left(y^{*}\right)$ для любого $y^{*} \in Y^{*}$. Кроме того, из неравенства

$$
\int_{0}^{1} u(\dot{y}(t)) d t+\int_{0}^{1} u^{*}\left(\dot{y}^{*}(t)\right) d t \geqslant \int_{0}^{1} \dot{y}(t) \dot{y}^{*}(t) d t=\left\langle y, y^{*}\right\rangle
$$

и определения (1.5) вытекает, что

$$
h^{*}\left(y^{*}\right)=\sup _{y \in Y}\left\{\left\langle y, y^{*}\right\rangle-h(y)\right\} \leqslant \int_{0}^{1} u^{*}\left(\dot{y}^{*}(t)\right) d t
$$

т.е.

$$
g^{*}\left(y^{*}\right) \leqslant h^{*}\left(y^{*}\right) \leqslant \int_{0}^{1} u^{*}\left(\dot{y}^{*}(t)\right) d t \quad \forall y^{*} \in Y^{*} .
$$

Теперь воспользуемся неравенством $u^{* *}(\alpha) \geqslant\left[(|\alpha|-1)_{+}\right]^{2} \forall \alpha \in \mathbb{R}$ или после взятия операции сопряжения

$$
u^{*}(\beta) \leqslant \beta^{2} / 4+|\beta| \quad \forall \beta \in \mathbb{R} .
$$

В силу последних двух неравенств сопряженньй функщионал $g^{*}\left(y^{*}\right)$ всюду конечен, а потому и непрерьвен на гильбертовом пространстве $Y^{*}=H_{0}^{1}(0,1)$ (см. [65, с. 23]). Это доказьвает, что $y_{0}(t) \equiv 0$ - производная по Гато функционала $g^{*}\left(y^{*}\right)$ в нуле. В то же время, как мы видели, $g^{* *}(0)=0$, хотя, очевидно, $g(0)=1$. 


\section{§1.2. Характеризация выпуклости в терминах гладкости}

Теорема 1.2.1. Пусть $Y$ - банахово (слабо секвенииально полное банахово) пространство и функция $g(y): Y \rightarrow \overline{\mathbb{R}}$ пн. снизу (слабо пн. снизу) на $Y$. Кроме того, пусть мнохсество $C:=\left\{y^{*} \in Y^{*}: \partial g^{*}\left(y^{*}\right) \neq \varnothing\right\}$ не пусто и сопряжсенная функиия $g^{*}$ дифференцируема по Фреше (Гато) на С. Тогда функция $g(y)$ выпукла [55].

ДокАЗАТЕЛЬСТвО. Пусть $D:=\left\{y=\nabla g^{*}\left(y^{*}\right), y^{*} \in C\right\}$ будет образоммножества $C$ при градиентном отображении $y=\nabla g^{*}\left(y^{*}\right)$. В обоих случаях это множество расположено в банаховом пространстве $Y$ (см. замечание к теореме 1.1.1), так что согласно теореме 1.1.1 равенство (1.1) справедливо на множестве D. Напомним, что [39], [45]

$$
y \in \partial g^{*}\left(y^{*}\right) \Longleftrightarrow y^{*} \in \partial g^{* *}(y)
$$

и

$$
\partial g^{*}\left(y^{*}\right)=\left\{\nabla g^{*}\left(y^{*}\right)\right\}, \quad y^{*} \in C .
$$

Поскольку субдифференциал $\partial g^{*}\left(y^{*}\right)$ пуст, если $y^{*} \neq C$, бисопряженная функция $g^{* *}(y)$ субдифференцируема в точке $y$, только если $y \in D$. По теореме Бренстеда и Рокафеллара [39], [73] для любой точки $y_{0} \in \operatorname{dom} g^{* *}$ существует последовательность $y_{k} \rightarrow Y_{0}$ такая, что

$$
\partial g^{* *}\left(y_{k}\right) \neq \varnothing, \quad k=1,2, \ldots, \quad g^{* *}\left(y_{0}\right)=\lim _{k \rightarrow \infty} g^{* *}\left(y_{k}\right)
$$

Поэтому $y_{k} \in D$ и двойственность (1.1) становится $g^{* *}\left(y_{k}\right)=g\left(y_{k}\right), k=1,2, \ldots$. В силу пн. снизу функции $g(y)$ отсюда получаем

$$
g^{* *}\left(y_{0}\right)=\varliminf_{k \rightarrow \infty} g^{* *}\left(y_{k}\right)=\varliminf_{k \rightarrow \infty} g\left(y_{k}\right) \geqslant g\left(y_{0}\right)
$$

для любого $y_{0} \in \operatorname{dom} g^{* *}$ (здесь мы также пользуемся тем фактом, что слабо пн. снизу функция является пн. снизу и в сильной топологии). Поскольку в любом случае $g^{* *}\left(y_{0}\right) \leqslant g\left(y_{0}\right)$, двойственность (1.1) имеет место на множестве $\operatorname{dom} g^{* *}$. Но когда $y_{0} \notin \operatorname{dom} g^{* *}$, тогда $g\left(y_{0}\right) \geqslant g^{* *}\left(y_{0}\right)=+\infty$, так что $g(y) \equiv g^{* *}(y)$, и следовательно, функция $g(y)$ вьпукла.

ЗАмЕчАнИя. Этот результат справедлив в произвольном банаховом пространстве не только в случае Фреше-, но и Гато-дифференцируемости, если дополнительно потребовать, чтобы производные сопряженной функции принадлежали этому пространству. Можно доказать, что в действительности сопряженный функционал к рассмотренному вьше функционалу Больца дифференцируем по Гато на всем пространстве, так что в теореме 1.2.1 тоже нельзя заменить производную по Фреше на производную по Гато, если полунепрерывность снизу имеет место лишш в нормированной, но не слабой топологии.

Здесь мы покажем, что в проблеме вьпуклости чебьшёвских множеств эта теорема дает такой результат: всякое слабо замкнутое чебышёвское множество в гильбертовом пространстве выпукло. В конечномерном случае этот факт сводится 
к известной теореме Т. Моцкина, общий результат доказан в работе Н.В. Ефимова, С.Б. Стечкина [15]; см. также [68], [94], [95]. Напомним, что множество $A$ назьвается чебышёвским, если для любой точки $x \in H$ сушествует ровно один элемент $y(x)$ такой, что

$$
|y(x)-x|=\rho(x, A):=\inf _{y \in A}|y-x|, \quad x \in H, \quad y(x) \in A .
$$

Теорема 1.2.2. Пусть $A$ - замкнутое множество в гильбертовом пространстве H. Множсество А выпукло в том и только том случае, когда квадрат расстояния $\rho^{2}(x, A)$ от произвольной точки $x$ до множества $A$ дифферениируем по фреше на $H$ [95].

ДокАЗАТЕльство. Введем функцию $g(y):=|y|^{2} / 2+\delta(y \mid A)[68]$, где $\delta(y \mid A):=0$, $y \in A, \delta(y \mid A):=+\infty, y \notin A,-$ индикаторная функция множества $A$. Легко заметить, что она пн. снизу, а сопряженная функция $g^{*}(x)$ может быть записана как

$$
g^{*}(x)=|x|^{2} / 2-\rho^{2}(x, A) / 2 .
$$

Поэтому, если функция $\rho^{2}(x, A) \Phi$ реше-дифференцируема на $H$, сопряженная функщия $g^{*}(x)$ также $\Phi$ реше-дифференцируема на $H$, и по теореме 1.2 .1 функция $g(y)$ выпукла. Тогда ее множество уровня

$$
A=\{y \in H: g(y)<+\infty\}
$$

тоже вьпукло. Наоборот, если множество $A$ вьпукло и замкнуто в гильбертовом пространстве $H$, то индикаторная функция $\delta(y \mid A)$ выпукла и пн. снизу. Следовательно, функция $\rho^{2}(x, A)$ Фреше-дифференцируема на $H$ (см. [39, с. 194]).

ТеОрема 1.2.3. Пусть А будет слабо замкнутым множеством в гильбертовом пространстве Н. Тогда следующие свойства әквивалентньи [55]:

а) множсество А вьипукло;

b) $A$ - чебишёвское множество;

c) функция $\rho^{2}(x, A)$ дифференцируема по Гато на $H$;

d) функция $\rho^{2}(x, A)$ дифференцируема по Фреше на $H$.

ДокАЗАТЕльСТво. Поскольку слабо замкнутые множества замкнуты и в нормированной топологии, свойства а) и d) эквивалентны по теореме 1.2.2. Когда множество $A$ слабо замкнуто и выпукло, множества уровня $\left\{y \in A:|x-y|^{2} \leqslant \alpha\right\}$ пн. снизу строго вьпуклой функции $|x-y|^{2}$ слабо компактны, так что а) влечет за собой b) по теореме Вейерштрасса. Мы собираемся показать, что b) влечет за собой с) и с) влечет за собой а).

Пусть $A$ - чебьшёвское множество в $H$ и $y(x): H \rightarrow A$ - метрическая проекция точки $x \in H$ на множество $A$, которая единственньм образом определяется равенством (2.2). Из тождества (2.3) следует, что функция $g^{*}(x)$ так же, как и липшицева функция $\rho(x, A)$, непрерывна на $H$. Пользуясь слабой замкнутостью множества $A$, легко показать, что метрическая проекция $y(x)$ локально ограничена и имеет слабо-сильно замкнутьй график. 
Ключом к доказательству теоремы 1.2.3 является замечание, что $y(x) \in \partial g^{*}(x)$ для всех $x \in H$. Действительно, из (2.3) и (2.2) следует, что $g^{*}(x)=\langle y(x), x\rangle-g(y(x))$, так что $g^{*}(x)+g^{* *}(y(x)) \leqslant\langle y(x), x\rangle$, что означает $y(x) \in \partial g^{*}(x)$ (cм. [19], [45]).

Все, что нам теперь нужно - это доказать равенства

$$
\partial g^{*}(x)=\left\{\nabla g^{*}(x)\right\}=\{y(x)\}, \quad x \in H .
$$

С этой целью для произвольного элемента $y \in \partial g^{*}(x)$ мы рассмотрим последовательность $x_{k}=x+(y-y(x)) / k$. Поскольку $x_{k} \rightarrow x$ при $k \rightarrow \infty$ и метрическая проекция локально ограничена, сушествует подпоследовательность $\left\{x_{k_{n}}\right\}$ такая, что $y\left(x_{k_{n}}\right)$ слабо сходится к некоторой точке $z$ при $n \rightarrow \infty$. Как было замечено вьше, метрическая проекция имеет слабо-сильно замкнутый график, поэтому $z=y(x)$.

Учитывая монотонность субдифференщиального отображения $\partial g^{*}(x)$, мы получаем, что векторы $y\left(x_{k_{n}}\right) \in \partial g^{*}\left(x_{k_{n}}\right)$ и $y \in \partial g^{*}(x)$ подчиняются неравенствам $\left\langle y\left(x_{k_{n}}\right)-y, x_{k_{n}}-x\right\rangle \geqslant 0$, откуда $\left\langle y\left(x_{k_{n}}\right)-y, y-y(x)\right\rangle \geqslant 0, n=1,2, \ldots$ Когда $n$ стремится к бесконечности, это дает $-|y(x)-y|^{2} \geqslant 0$, ибо $y\left(x_{k_{n}}\right)$ слабо сходится к $y(x)$. Иными словами, $y=y(x)$ для любых векторов $x \in H$ и $y \in \partial g^{*}(x)$.

Таким образом, субдифференциальное отображение $\partial g^{*}(x)=\{y(x)\}$ однозначно. Как мы заметили, сопряженная функщия $g^{*}(x)$ непрерьвна. Поэтому она Гато-дифференцируема на $H[65]$. В силу тождества (2.3) это относится и к функции $\rho^{2}(x, A)$. Это доказьвает, что с) следует из b).

Наконец, если функция $\rho^{2}(x, A)$ Гато-дифференцируема на $H$, то это относится и к функции $g^{*}(x)$. Поскольку множество $A$ слабо замкнуто, функция $g(y)$ слабо пн. снизу и по теореме 1.2.1 она вьпукла. Следовательно, ее множество уровня (2.4) также вьпукло. Таким образом, а) следует из с).

Теперь мы приведем достаточные условия выпуклости множества $A$ в терминах гладкости его опорной функции

$$
\delta^{*}\left(y^{*} \mid A\right)=\sup _{y \in A}\left\langle y^{*}, y\right\rangle
$$

Теорема 1.2.4. Пусть произвольное множество А будет замкнуто (слабо замкнуто) в банаховом пространстве (слабо секвенциально полном банаховом пространстве) $Y \neq \mathbb{R}^{1}$, а его опорная функция $\delta^{*}\left(y^{*} \mid A\right) \not \equiv+\infty$ и дифференцируема по фреше (Гато) во всякой точке $y^{*} \neq 0$, в которой $\partial \delta^{*}\left(y^{*} \mid A\right) \neq \varnothing$. Тогда если замыкание его выпуклой оболочки $\overline{\operatorname{conv}} A$ имеет непустую внутренность, то справедливо равенство [56]

$$
\overline{\operatorname{conv}} A=(A+A) / 2 .
$$

ДокАЗАТЕЛЬСТВо. Возьмем любую граничную точку $y_{0} \in \overline{\operatorname{conv}} A$. Поскольку это множество имеет непустую внутренность, то по теореме отделимости найдется вектор $y_{0}^{*} \neq 0$ такой, что

$$
\left\langle y, y_{0}^{*}\right\rangle \leqslant\left\langle y_{0}, y_{0}^{*}\right\rangle \quad \forall y \in \overline{\operatorname{conv}} A
$$


Поэтому $y_{0} \in \partial \delta^{*}\left(y_{0}^{*} \mid \overline{\operatorname{conv}} A\right)$ (см. [19, с. 209]), так что по условию теоремы вектор $y_{0} \in Y$ является производной (Гато) Фреше опорной функции

$$
\delta^{*}\left(y^{*} \mid A\right)=\delta^{*}\left(y^{*} \mid \overline{\operatorname{conv}} A\right)
$$

в точке $y_{0}^{*} \in Y^{*}$. Применим теорему 1.1 .1 к (слабо) пн. снизу функции $g(y)=\delta(y \mid A)$, сопряженная к которой равна опорной функции множества $A$. По этой теореме

$$
\delta\left(y_{0} \mid A\right)=\delta^{* *}\left(y_{0} \mid A\right)=\delta\left(y_{0} \mid \overline{\operatorname{conv}} A\right)=0,
$$

что означает $y_{0} \in A$. Таким образом, все граничные точки замькания вьпуклой оболочки множества $A$ принадлежат самому множеству $A$.

Теперь возьмем любую точку $y_{0} \in \operatorname{int} \overline{\operatorname{conv}} A$. Поскольку $Y \neq \mathbb{R}^{1}$, она принадлежит некоторому двумерному сечению $B$ множества $\overline{\mathrm{conv}} A$, которое тоже вьпукло и замкнуто и содержит точку $y_{0}$ внутри себя. Без ограничения обшности рассуждения можно считать, что $y_{0}=0$ и это сечение лежит на плоскости $\mathbb{R}^{2}$.

Поскольку $0 \in \operatorname{int} B$, то калибровочная функция $k(x)$ сечения $B$ конечна при всех $x \in \mathbb{R}^{2}$. Поэтому вьпуклая функция $k(x)$ непрерьвна. Покажем, что $k(c)=k(-c)$ для некоторого вектора $c \in \mathbb{R}^{2}, c \neq 0$. Для этого введем функции $r(u):=k(\cos u, \sin u)$ и $S(u):=r(u+\pi)-r(u)$. Функция $S(u)$ непрерьвна и меняет знак на отрезке $[0, \pi]$, ибо $S(\pi):=r(2 \pi)-r(\pi)=r(0)-r(\pi):=-S(0)$. Поэтому найдется угол $u$ такой, что $S(u)=0$, т.е. $k(c)=k(-c)$ для вектора $c=(\cos u, \sin u)$.

Теперь заметим, что $k(c) \neq 0$, ибо иначе сечение $B$ целиком содержало бы прямую $\left\{\alpha c \mid \alpha \in \mathbb{R}^{1}\right\}$. Тогда само множество $\overline{\operatorname{conv}} A$ тоже содержало бы прямую $\left\{\alpha z+y_{0} \mid\right.$ $\left.\alpha \in \mathbb{R}^{1}\right\}$, и поэтому опорная функщия (2.7) была бы конечной только в гиперплоскости $\left\langle z, y^{*}\right\rangle=0$, что противоречит дифференцируемости этой функци в точке $y_{0}^{*} \in Y$. Таким образом, $k(c)=k(-c)>0$ и поэтому $0 \in \mathbb{R}^{2}-$ середина отрезка с концами в точках $\pm c / k( \pm c)$, лежаших на границе сечения $B$, а потому и на гранище самого множества $\overline{\operatorname{conv}} A$.

Окончательно, если $y_{0}-$ граничная точка множества $\overline{\operatorname{conv}} A$, то $y_{0}=\left(y_{0}+y_{0}\right) / 2$ с вектором $y_{0} \in A$, а если $y_{0}-$ внутренняя точка $\overline{\operatorname{conv}} A$, то $y_{0}=\left(y_{1}+y_{2}\right) / 2$ с векторами $y_{i} \in A, i=1,2$, лежащими на границе множества $\overline{\operatorname{conv}} A$.

СлЕДСТВИЕ 1.2.5. Если множество $A \subset \mathbb{R}^{n}$ замкнуто, а субдифференииальи его опорной функции $\delta^{*}\left(y^{*} \mid A\right)$ при $y^{*} \neq 0$ содержат не более одного әлемента, то при $n \neq 1$ и $\delta^{*}\left(y^{*} \mid A\right) \not \equiv+\infty$ имеет место представление (2.6).

ДокАЗАТЕльство. В этом случае множество $\overline{\operatorname{conv}} A$ либо состоит из одной точки, либо имеет непустую внутренность, ибо в противном случае это множество лежит в некоторой гиперплоскости с нормалью $y_{0}^{*} \neq 0$, и поэтому субдифференциал $\partial \delta^{*}\left(y_{0}^{*} \mid A\right)=\overline{\mathrm{conv}} A$ должен состоять из одной точки. Кроме того, опорная функция дифференщируема во всякой точке $y^{*} \neq 0$, в которой $\partial \delta^{*}\left(y^{*} \mid A\right) \neq \varnothing($ см. [45]).

СлЕДСТВИЕ 1.2.6. Если множсество $A \subset \mathbb{R}^{n}$ гомеоморфно некоторому выпуклому компакту в $\mathbb{R}^{n}$, а его опорная функция дифференцируема всюду, кроме нуля, то әто множество выпукло.

Это частный случай результатов Либермана (см. [10], [101]).

В заключение на еще одном примере мы покажем, что теорема 1.2.1 является довольно гибким инструментом исследования вьпуклости множеств. 
ТеОрема 1.2.7. Пусть функиия $f(y): \mathbb{R}^{n} \rightarrow \overline{\mathbb{R}}$ выпукла и пн. снизу на всем пространстве и дифференцируема на выпуклом компакте $C$. Тогда выпуклость множества $A=\left\{y^{*}=\nabla f(y), y \in C\right\}$ әквивалентна дифференцируемости функuиu

$$
f_{c}(y):=\sup _{x \in C}\{f(x)+\langle\nabla f(x), y-x\rangle\} .
$$

ДоказАтельство. Рассмотрим функцию $g\left(y^{*}\right):=f^{*}\left(y^{*}\right)+\delta\left(y^{*} \mid A\right)$, которая пн. снизу, ибо градиентное отображение $\nabla f(y)$ непрерывно [45] и переводит компакт $C$ в компакт $A$. В силу тождества [19], [45]

$$
f^{*}\left(y^{*}\right)+f(y)=\left\langle y^{*}, y\right\rangle, \quad y^{*}=\nabla f(y), \quad y \in C,
$$

сопряженная функция равна

$$
g^{*}(y)=\sup _{y^{*} \in A}\left\{\left\langle y, y^{*}\right\rangle-f^{*}\left(y^{*}\right)\right\}=f_{c}(y) .
$$

Если она всюду дифференщируема, то по теореме 1.2.1 исходная функция $g\left(y^{*}\right)$ выпукла, а потому будет вьпукла и ее область определения $A$.

Наоборот, пусть компактное множество $A$ выпукло. Пользуясь дифференцируемостью функции $f(y)$ на множестве $C$, нетрудно доказать, что сопряженная к ней функция строго выпукла на множестве $A$. Тогда при каждом $y \in \mathbb{R}^{n}$ максимум в (2.8) достигается ровно на одном элементе $y^{*}=y^{*}(y) \in A$. По теореме о субдифференциале максимума семейства вьпуклых функций (см. [19, с. 213]) $\partial g^{*}(y)=\left\{y^{*}(y)\right\}$ для любого $y$, т.е. функция $g^{*}(y) \equiv f_{c}(y)$ всюду дифференцируема.

ЗАмечАнИЕ. Функция $f_{c}(y)$ является наименьшей вьпуклой полунепрерьвной снизу функцией, совпадающей с $f(y)$ на множестве $C$ (это вытекает из неравенства $f(y) \geqslant f(x)+\langle\nabla f(x), y-x\rangle)$.

\section{§1.3. Теоремы о минимаксе}

Теорема Дж. фон Неймана [82] и ее обобщения [39] утверждают, что если функция $L(x, y)$ выпукла по $x$ на множестве $A$ при каждом фиксированном $y \in Y$ и вогнута по $y$ на множестве $Y$ при каждом фиксированном $x \in A$, то при некоторых топологических предположениях на лагранжиан $L(x, y)$ справедливо минимаксное соотношение (3.2). Здесь мы покажем, что условие вогнутости можно заменить на условие дифференцируемости функции

$$
\varphi(x):=\sup _{y \in Y} L(x, y): X \rightarrow \overline{\mathbb{R}}
$$

в точке ее минимума на множестве $A$. По-видимому, впервые этот факт был обнаружен С. Верду [84] и затем был переоткрыт И. Ф. Пинелисом [40], [41] и автором [47], [49]. В настоящей работе мы приводим некоторые улучшения этих результатов, основанные на следуюшей лемме.

Лемма 1.3.1. Пусть функиии $\psi_{\alpha}(t): \mathbb{R} \rightarrow \overline{\mathbb{R}}$ выпукльи и их верхняя грань $\psi(t):=$ $\sup _{\alpha} \psi_{\alpha}(t)$ дифференцируема в точке $\tau$. Тогда все функиии $\psi_{\alpha}(t)$ такие, что $\psi_{\alpha}(\tau)=\psi(\tau)$, тоже дифференцируемы в точке $\tau$ и $\psi_{\alpha}^{\prime}(\tau)=\psi^{\prime}(\tau)$. 
ДокАЗАТЕЛЬСТВО. Поскольку функция $\psi(\tau)$ конечна в окрестности точки $\tau$, то все функции $\psi_{\alpha}(\tau)$ тоже конечны в этой окрестности. Очевидно, что если $\psi_{\alpha}(\tau)=$ $\psi(\tau)$, то

$$
\psi^{\prime}(\tau)=\psi^{\prime}(\tau ; 1) \geqslant \psi_{\alpha}^{\prime}(\tau ; 1), \quad-\psi^{\prime}(\tau)=\psi^{\prime}(\tau ;-1) \geqslant \psi_{\alpha}^{\prime}(\tau ;-1) .
$$

Складывая эти неравенства, получим $0 \geqslant \psi_{\alpha}^{\prime}(\tau ; 1)+\psi_{\alpha}^{\prime}(\tau ;-1) \geqslant \psi_{\alpha}^{\prime}(\tau ; 0)=0$, ибо производная $\psi_{\alpha}^{\prime}(\tau ; \cdot)$ выпукла. Таким образом, правая и левая производные функции $\psi_{\alpha}(t)$ в точке $\tau$ равны $\psi^{\prime}(\tau)$.

Tеорема 1.3.2. Пусть функиия $L(x, y): X \times Y \rightarrow \overline{\mathbb{R}}$ выпуклла по $x$ при фиксированном $y \in Y$ на линейном пространстве $X$ и множество $A \subset X$ выпукло. Пусть $x_{*} \in A-$ точка минимума функции (3.1) на множсестве А, причем при $x=x_{*}$ максимум (3.1) достигается на некотором әлементе $y_{*} \in Y$. Если функиия (3.1) имеет двусторонние производные $\varphi^{\prime}\left(x_{*} ; x-x_{*}\right)$ в любом допустимом направлении $x-x_{*}, x \in A$, то $\left(x_{*}, y_{*}\right)$ - седловая точка функиии $L(x, y)$ на множестве $A \times Y$ и

$$
\min _{x \in A} \sup _{y \in Y} L(x, y)=\max _{y \in Y} \inf _{x \in A} L(x, y) .
$$

ДокАЗАТЕЛЬСТво. Функщия (3.1) достигает своего минимума на множестве $A$ в точке $x_{*} \in A$, поэтому $\varphi\left(\lambda x+(1-\lambda) x_{*}\right) \geqslant \varphi\left(x_{*}\right)$ для всех $x \in A, \lambda \in(0,1)$, и, следовательно, $\left(\varphi\left(x_{*}+\lambda\left(x-x_{*}\right)\right)-\varphi\left(x_{*}\right)\right) / \lambda \geqslant 0$. При $\lambda \rightarrow 0$ получаем из этого неравенства

$$
\varphi^{\prime}\left(x_{*} ; x-x_{*}\right) \geqslant 0 \quad \forall x \in A .
$$

Поскольку функщия (3.1) имеет двусторонние производные (3.3) и по условию

$$
L\left(x_{*}, y_{*}\right)=\max _{y \in Y} L\left(x_{*}, y\right),
$$

то по лемме 1.3 .1$, примененной к функциям $\psi_{y}(t)=L\left(x_{*}+t\left(x-x_{*}\right), y\right)$ в точке $\tau=0$, производная функции $L\left(\cdot, y_{*}\right)$ в точке $x_{*}$ по любому направлению $x-x_{*}, x \in A$, равна соответствующей производной по направлению функции $\varphi(x)$.

В силу этого и вьпуклости лагранжиана $L\left(\cdot, y_{*}\right)$ имеем

$$
L\left(x, y_{*}\right)-L\left(x_{*}, y_{*}\right) \geqslant \varphi^{\prime}\left(x_{*} ; x-x_{*}\right), \quad x \in A,
$$

что ввиду (3.3) преобразуется к

$$
L\left(x_{*}, y_{*}\right)=\min _{x \in A} L\left(x, y_{*}\right)
$$

Соотношения (3.4), (3.6) означают, что $\left(x_{*}, y_{*}\right)$ - седловая точка лагранжиана $L(x, y)$ на множестве $A \times Y$, и поэтому справедлива двойственность (3.2) [65]. 
ТеОрема 1.3.3. Пусть функиия $L(x, y): X \times Y \rightarrow \overline{\mathbb{R}}$ выпукла по $x$ на линейном пространстве $X$ и полунепрерывна сверху по у на компактном топологическом пространстве $Y$, и пусть $x_{*} \in A-$ точка минимума функции (3.1) на выпуклом множестве $A \subset X$. Тогда двойственность (3.2) справедлива при выполнении любого из следующих условии:

а) для фиксированного $x \in A$ функиии $L\left(\cdot, y_{*}\right)$ имеют двусторонние производные в точке $x_{*}$ в направлении $x-x_{*}$, которые совпадают для всех әлементов $y_{*} \in Y$, дающих максимум в (3.4);

b) функции $L\left(\cdot, y_{*}\right)$ совпадают для всех әлементов $y_{*} \in Y$, дающих максимум в (3.4).

ДокАЗАТЕЛЬСТво. Воспользуемся равенством [19], [43]

$$
\psi^{\prime}(\tau)=\max _{\alpha}\left\{\psi_{\alpha}^{\prime}(\tau) \mid \psi_{\alpha}(\tau)=\psi(\tau)\right\} .
$$

В случае а) функция (3.1) имеет двусторонние производные $\psi^{\prime}\left(x_{*} ; x-x_{*}\right)$ в любом направлении $x-x_{*}, x \in A$, и теорема 1.3 .3 следует из теоремы 1.3 .2 . В случае b) мы получаем из $(3.7)$, что производные по направлению функции $L\left(\cdot, y_{*}\right)$ равны соответствующим производным функции $\varphi(x)[41]$, что дает неравенство $(3.5)$, которое использовалось в доказательстве теоремы 1.3.2.

СлЕДСТВИЕ 1.3.4. Пусть функиия $L(x, y): X \times Y \rightarrow \overline{\mathbb{R}}$ выпукла по х на линейном пространстве $X$ и полунепрерывна сверху по у на компактном топологическом пространстве $Y$, и пусть $x_{*} \in A$ - точка минимума функции (3.1) на выпуклом множсестве $A \subset X$. Если максимум (3.4) достигается на единственном әлементе $y_{*} \in Y$, то справедлива двойственность (3.2) [40].

В настоящее время известны два подхода к теории двойственности, основанные на теории сопряженных функщий и на теоремах о минимаксе, которые в вьпуклом случае почти идентичны (см. [65], а также доказательство леммы 1.4.1). Как видно из $\S \S 1.1,1.3$, в невьпуклом случае эти подходы уже не так близки.

\section{$\S$ 1.4. Сопряженная функция к максимуму семейства квадратичных форм}

Пусть $C_{i}, i=1, \ldots, k$, будут компактными подмножествами евклидова $n$-мерного пространства $\mathbb{R}^{n}$, а $\mathscr{D}$ будет компактным множеством неотрицательно определенных матриц $D$ порядка $n$. Рассмотрим функцию

$$
2 D(x):=\max _{D \in \mathscr{D}, c_{i} \in C_{i}}\langle D x, x\rangle+\sum_{i=1}^{k}\left\langle c_{i}, x\right\rangle^{2} .
$$

Без потери обшности можно предположить, что множества $C_{i}$ центрально-симметричны и все множества $\mathscr{D}, C_{i}, i=1, \ldots, k$, выпуклы (иначе нужно симметризовать множества $C_{i}$ и заменить все эти множества на их вьпуклые оболочки). Введем множество

$$
\mathscr{K}=\left\{K=D+\sum_{i=1}^{k} c_{i} c_{i}^{T} \mid D \in \mathscr{D}, c_{i} \in C_{i}, i=1, \ldots, k\right\}
$$


неотрицательно определенных матриц $K$ порядка $n$. Тогда

$$
2 D(x)=\max _{K \in \mathscr{K}}\langle K x, x\rangle .
$$

Пусть $\mathscr{R}(K):=\left\{y=K x: x \in \mathbb{R}^{n}\right\}$ и $\mathscr{N}(K):=\left\{x \in \mathbb{R}^{n}: K x=0\right\}$ будут образом и ядром матрицы $K \geqslant 0$ соответственно. Как известно (см. [45, с. 125]), сопряженная функция к квадратичной форме $g(x):=\langle K x, x\rangle / 2$, равна

$$
g^{*}(y)=\left\langle K^{+} y, y\right\rangle / 2
$$

где $K^{+}$обозначает псевдообратную матрищу, причем по соглашению $\left\langle K^{+} y, y\right\rangle:=+\infty$ при $y \notin \mathscr{R}(K)$. Чтобы обобшить этот результат, нам нужна следующая

Лемма 1.4.1. Пусть D будет неотрицательно определенной матрицей порядка п и все множества $C_{i}$ строго выпукль. Тогда

$$
f(y):=\inf _{c_{i} \in C_{i}}\left\langle\left(D+\sum_{i=1}^{k} c_{i} c_{i}^{T}\right)^{+} y, y\right\rangle / 2
$$

будет выпуклой функиией на $\mathbb{R}^{n}$.

1-Е ДОКАЗАТЕЛЬСТВО. ОПорные функции $\delta^{*}\left(x \mid C_{i}\right)$ строго вьпуклых множеств $C_{i}$ дифференцируемы всюду, кроме нуля. Поэтому функция

$$
2 D(x)=\langle D x, x\rangle+\sum_{i=1}^{k} \delta^{* 2}\left(x \mid C_{i}\right)
$$

тоже всюду дифференцируема (ее градиент в нуле равен нулю, ибо $\delta^{* 2}(x \mid C) \leqslant$ $M^{2}|x|^{2}$, если $|c| \leqslant M$ для любого вектора $c \in C$ ). Проверим, что функция (4.5) сопряжена к функции $f(y)$ :

$$
\begin{aligned}
f^{*}(x) & =\sup _{y}\left\{\langle x, y\rangle-\inf _{c_{i} \in C_{i}}\left\langle K^{+} y, y\right\rangle / 2\right\} \\
& =\sup _{c_{i} \in C_{i}} \sup _{y}\left\{\langle x, y\rangle-\left\langle K^{+} y, y\right\rangle / 2\right\} \\
& \text { по (4.3a) и (1.1) } \\
& =\sup _{c_{i} \in C_{i}}\langle K y, y\rangle / 2=D(x) .
\end{aligned}
$$

По теореме о маргинальном значении (см. [39, следствие 3.22]) функция $f(y)$ пн. снизу, ибо функция (4.3а) пн. снизу по обеим переменньм $K$ и $y$ в совокупности и отображение

$$
K=D+\sum_{i=1}^{k} c_{i} c_{i}^{T}
$$

непрерьвно по переменным $c_{i}$ на компактных множествах $C_{i}, i=1, \ldots, k$. Таким образом, функция $f(y)$ вьпукла по теореме 1.2.1. 
2-Е доКАЗАТЕЛЬСТво. Введем лагранжиан

$$
L(x, c):=\left\langle\left(D+\sum_{i=1}^{k} c_{i} c_{i}^{T}\right) x, x\right\rangle / 2-\langle x, y\rangle,
$$

являюшийся вьпукльм по $x$ на $\mathbb{R}^{n}$ и пн. сверху по переменным $c=\left(c_{1}, \ldots, c_{k}\right)$ на декартовом произведении $C_{1} \times \cdots \times C_{k}$. С учетом (4.5) функция (3.1) принимает вид

$$
\varphi(x)=\sup _{c_{i} \in C_{i}} L(x, c)=D(x)-\langle x, y\rangle .
$$

Она всюду дифференцируема, и мы далее покажем, что ее минимум достигается в некоторой точке $x_{0}$. Поэтому по теореме 1.3 .2

$$
\begin{aligned}
\min _{x \in \mathbb{R}^{n}} \varphi(x) & =\max _{c_{i} \in C_{i}} \inf _{x \in \mathbb{R}^{n}} L(x, c) \\
\text { по (4.3a) } & =\max _{c_{i} \in C_{i}}\left\{-\left\langle K^{+} y, y\right\rangle / 2\right\}=-f(y) .
\end{aligned}
$$

Чтобы доказать, что точка минимума $x_{0}$ функции $\varphi(x)$ действительно существует, достаточно заметить, что $x_{0} \in \partial D^{*}(y)$ (см. [39, теорема 23.5]). Поскольку функция $D(x)$ однородна степени два, то сопряженная функция $D^{*}(y)$ тоже однородна степени два (см. [39, следствие 15.3.1]), так что она конечна на линейном пространстве в $\mathbb{R}^{n}$. Поэтому она субдифференцируема в каждой точке $y$, где она конечна [39]:

$$
x_{0} \in \partial D^{*}(y) \neq \varnothing, \text { если } D^{*}(y) \neq+\infty .
$$

Теперь (4.8) становится $D^{*}(y)=f(y)$, если $D^{*}(y) \neq+\infty$. Но когда $D^{*}(y)=+\infty$, это тоже верно, ибо minmax $\geqslant \operatorname{maxmin.~Таким~образом,~функция~} f(y) \equiv D^{*}(y)$ выпукла, так как $D^{*}(y)$ является верхней гранью линейных функций.

Следующий результат будет играть ключевую роль в дальнейшем.

ТеОРема 1.4.2. Пусть $\mathscr{D}$ будет компактным выпуклым множеством неотрицательно определенных матриц порядка $n, C_{i}, i=1, \ldots, k,-$ компактные выпуклье и центрально симметричные множества в $\mathbb{R}^{n}$ и

$$
\mathscr{L}:=\bigcap_{K \in \mathscr{K}} \mathscr{N}(K)=\left\{x \in \mathbb{R}^{n} \mid D(x)=0\right\}
$$

будет пересечением ядер всех матрич из мнохсества (4.2). Тогда сопряжсеная $\kappa$ функции (4.3) имеет вид

$$
D^{*}(y)= \begin{cases}\min _{K \in \mathscr{K}}\left\langle K^{+} y, y\right\rangle / 2, & y \in \mathscr{L}^{\perp}, \\ +\infty, & y \notin \mathscr{L}^{\perp} .\end{cases}
$$

Для фиксированного вектора $y \in \mathscr{L}^{\perp}$ матрица $K_{y} \in \mathscr{K}$ тогда и только тогда будет решением вспомогательной задачи (4.10), когда

$$
\left\langle K_{y} x_{y}, x_{y}\right\rangle=\max _{K \in \mathscr{K}}\left\langle K x_{y}, x_{y}\right\rangle
$$

для вектора $x_{y}$ такого, что $K_{y} x_{y}=y$. В этом случае

$$
D^{*}(y)=D\left(x_{y}\right), \quad x_{y} \in \partial D^{*}(y) .
$$


ДокАЗАТЕЛЬСтво. Сначала рассмотрим одноточечное множество $\mathscr{D}=\{D\}$ и любые множества $C_{i}$. Тогда существуют непрерывные многозначные отображения $\alpha \rightarrow C_{i}(\alpha), \alpha \in[0,1]$, с компактными, строго вьпукльми, центрально симметричными значениями $C_{i}(\alpha) \supset C_{i}, \alpha \neq 0$, такие, что $C_{i}(0)=C_{i}, i=1, \ldots, k$. Определим функции $f_{\alpha}(y)$ заменой множеств $C_{i}$ в $(4.4)$ на множества $C_{i}(\alpha)$. По теореме о маргинальном значении (см. [39, предложение 3.21]) $\underline{\lim }_{\alpha \rightarrow 0} f_{\alpha}(y) \geqslant f(y)$. С другой стороны, $\overline{\lim }_{\alpha \rightarrow 0} f_{\alpha}(y) \leqslant f(y)$, ибо $C_{i}(\alpha) \supset C_{i}$. Как было показано в лемме 1.4.1, функции $f_{\alpha}(y)$ вьпуклы и пн. снизу для $\alpha \neq 0$, поэтому их предел $f(y)$ тоже вьпуклый и пн. снизу. Следовательно, в силу (1.1) и (4.6)

$$
f(y)=\left(f^{*}\right)^{*}(y)=D^{*}(y):=\sup _{x}\left\{\langle x, y\rangle-\left[\langle D x, x\rangle+\sum_{i=1}^{k} \delta^{* 2}\left(x \mid C_{i}\right)\right] / 2\right\} .
$$

Таким образом, функция $f(D, y):=f(y)$ есть верхняя грань линейных функций. Поэтому она вьпукла на множестве $\mathscr{D} \times \mathbb{R}^{n}$. Следовательно, ее минимум

$$
\varphi(y):=\inf _{D \in \mathscr{D}} f(D, y)=\inf _{K \in \mathscr{K}}\left\langle K^{+} y, y\right\rangle / 2
$$

на компактном, выпуклом множестве $\mathscr{D}$ является выпуклой функцией [45]. Функция $\varphi(y)$ пн. снизу, ибо функция $f(D, y)$ пн. снизу по обеим переменным $D$ и $y$ и отображение (4.7) непрерьвно по переменной $D$ на компактном множестве $\mathscr{D}$. По той же причине минимум вверху достигается всякий раз, когда он конечен. Вновь легко проверяется, что $\varphi^{*}(x)=D(x)$ (как определено в $(4.1)$ ), откуда $D^{*}(y)=\varphi^{* *}(y)=\varphi(y)$, как и требовалось в (4.10).

Теперь заметим, что $\left\langle K^{+} y, y\right\rangle \neq+\infty$, только если $y \in \mathscr{R}(K)$. Поэтому $\operatorname{dom}\left(D^{*}\right):=$ $\left\{y \mid D^{*}(y)<+\infty\right\}=\bigcup_{K \in \mathscr{K}} \mathscr{R}(K)$. Как мы знаем, это множество является линейным пространством в $\mathbb{R}^{n}$, поэтому [45]

$$
\begin{aligned}
\operatorname{dom}\left(D^{*}\right) & =\left[\operatorname{dom}\left(D^{*}\right)\right]^{* *}=\left[\left(\bigcup_{K \in \mathscr{K}} \mathscr{R}(K)\right)^{*}\right]^{*}=\left[\bigcap_{K \in \mathscr{K}} \mathscr{R}^{*}(K)\right]^{*} \\
& =\left[\bigcap_{K \in \mathscr{K}} \mathscr{R}^{\perp}(K)\right]^{*}=\left[\bigcap_{K \in \mathscr{K}} \mathscr{N}(K)\right]^{*}=\mathscr{L}^{*}=\mathscr{L}^{\perp} .
\end{aligned}
$$

Это завершает доказательство (4.10).

Если $y \in \mathscr{L}^{\perp}$, то согласно (4.9) существует вектор $x_{y} \in \partial D^{*}(y)$. Это эквивалентно равенству

$$
D\left(x_{y}\right)+D^{*}(y)=\left\langle x_{y}, y\right\rangle .
$$

Поскольку функция $\left\langle K^{+} y, y\right\rangle$ пн. снизу на компактном множестве $\mathscr{K}$, сушествует матрица $K_{y}$ такая, что

$$
\left\langle K_{y}^{+} y, y\right\rangle / 2=D^{*}(y), \quad y \in \mathscr{L}^{\perp} .
$$


Тогда (4.3) и (4.13) означают, что

$$
\left\langle K_{y} x_{y}, x_{y}\right\rangle / 2+\left\langle K_{y}^{+} y, y\right\rangle / 2 \leqslant\left\langle x_{y}, y\right\rangle .
$$

Но это возможно только при $K_{y} x_{y}=y$.

Чтобы доказать (4.11), достаточно заметить, что если бы левая часть (4.11) была меньше, чем ее правая часть, мы бы получили строгое неравенство в $(4.15)$, противоречие. Первое тождество в (4.12) следует из (4.14) и (4.11):

$$
\begin{aligned}
2 D^{*}(y) & =\left\langle K_{y}^{+} y, y\right\rangle=\left\langle K_{y}^{+} K_{y} x_{y}, K_{y} x_{y}\right\rangle \\
& =\left\langle K_{y} K_{y}^{+} K_{y} x_{y}, x_{y}\right\rangle=\left\langle K_{y} x_{y}, x_{y}\right\rangle=2 D\left(x_{y}\right), \quad y \in \mathscr{L}^{\perp} .
\end{aligned}
$$

Наоборот, если (4.11) вьполнено для вектора $x_{y}$ такого, что $K_{y} x_{y}=y$, то по теореме о субдифференциале максимума семейства вьпуклых функций [19] $y=K_{y} x_{y}$ $\in \partial D\left(x_{y}\right)$ и, следовательно, $x_{y} \in \partial D^{*}(y)$. Поэтому из (4.13) и (4.11) получаем

$$
D^{*}(y)=\left\langle x_{y}, y\right\rangle-D\left(x_{y}\right)=\left\langle x_{y}, y\right\rangle-\left\langle K_{y} x_{y}, x_{y}\right\rangle / 2=\left\langle K_{y} x_{y}, x_{y}\right\rangle / 2 .
$$

Следовательно, $K_{y}$ - решение вспомогательной задачи (4.10).

ТеОрема 1.4.3 [87]. Если все матрицы из множества (4.2) положительно определены, то функция (4.3) строго выпукла, а ее сопряжсенная непрерывно дифференцируема, причем $\nabla D^{*}(y)=x_{y}=K_{y}^{-1} y$.

СлЕДСТВИЕ 1.4.4. Если ядро $\mathscr{L}$ в (4.9а) равно нулю, то

$$
\|x\|_{\mathscr{K}}:=\max _{K \in \mathscr{K}}\langle K x, x\rangle^{1 / 2} \quad u \quad\|y\|_{\mathscr{K}}^{0}=\min _{K \in \mathscr{K}}\left\langle K^{+} y, y\right\rangle^{1 / 2}
$$

являются двойственными нормами в $\mathbb{R}^{n}$.

Это вытекает непосредственно из [39, следствие 15.3.2].

СлЕДСТВИЕ 1.4.5. Оббединение эллипсоидов

$$
\bigcup_{K \in \mathscr{K}}\left\{y \in \mathbb{R}^{n}:\left\langle K^{+} y, y\right\rangle \geqslant 1\right\}
$$

выпукло и компактно, если $\mathscr{L}=\{0\}$.

В самом деле, его можно записать как множество уровня нормы $\left\{y:\|y\|_{\mathscr{K}}^{0} \leqslant 1\right\}$.

СлЕДСТВИЕ 1.4.6. Опорная функиия $\delta^{*}(y \mid E)$ пересечения

$$
E:=\bigcap_{K \in \mathscr{K}}\left\{x \in \mathbb{R}^{n}:\langle K x, x\rangle \leqslant 1\right\}
$$

эллипсоидов равна

$$
\delta^{*}(y \mid E)=\min _{K \in \mathscr{K}}\left\langle K^{+} y, y\right\rangle^{1 / 2} .
$$

Это следует из представления $E=\{x: D(x) \leqslant 1 / 2\}$ и [45, теорема 13.5]. 
СЛЕДСТВИЕ 1.4.7. Пусть все матрицы $D \in \mathscr{D}$ невырожденны, и пусть

$$
2 D(x)=\max _{D \in \mathscr{D}}\langle D x, x\rangle+\|x\|^{2}, \quad\|x\|:=\max _{c \in C}\langle x, c\rangle .
$$

Тогда

$$
\begin{gathered}
2 D^{*}(y)=\min _{D \in \mathscr{D}, c \in C}\left\{\left\langle D^{-1} y, y\right\rangle-\frac{\left\langle D^{-1} y, c\right\rangle^{2}}{1+\left\langle D^{-1} c, c\right\rangle}\right\}, \\
\nabla D^{*}(y)=x_{y}=D_{y}^{-1}\left(y-\lambda c_{y}\right),
\end{gathered}
$$

где $D_{y} \in \mathscr{D}, c_{y} \in C$ и $\lambda \geqslant 0$ удовлетворяют условиям

$$
\begin{gathered}
\left\langle D_{y} x_{y}, x_{y}\right\rangle=\max _{D \in \mathscr{D}}\left\langle D x_{y}, x_{y}\right\rangle, \quad c_{y} \in \partial\left\|x_{y}\right\|, \\
\lambda=\left\|x_{y}\right\| .
\end{gathered}
$$

ДоКАЗАТЕЛЬСТво. В силу (4.7)

$$
K^{-1}=D^{-1}-D^{-1} c\left(D^{-1} c\right)^{T} /\left(1+\left\langle D^{-1} c, c\right\rangle\right),
$$

что влечет за собой (4.18). Тогда правая часть (4.12) принимает вид (4.19), где $\lambda$ обозначает число

$$
\lambda=\left\langle D_{y}^{-1} c_{y}, y\right\rangle /\left(1+\left\langle D_{y}^{-1} c_{y}, c_{y}\right\rangle\right)
$$

Далее, в случае (4.17) уравнение (4.11) сводится к (4.20), так что остается доказать (4.21). Для этого воспользуемся равенством $\left\|x_{y}\right\|=\left\langle c_{y}, x_{y}\right\rangle$ [45]. После простого вычисления, использующего (4.19) и (4.23), последняя величина принимает вид (4.23).

СлеДСтвиЕ 1.4.8. Если $2 D(x)=|x|^{2}+\|x\|^{2}$, mo

$$
2 D^{*}(y)=|y|^{2}-\max _{c \in C} \frac{\langle y, c\rangle^{2}}{1+|c|^{2}}, \quad \nabla D^{*}(y)=x_{y}=y-\lambda c_{y},
$$

где величины $c_{y}$ и $\lambda$ удовлетворяют условиям (4.20), (4.21).

Результаты $\$ 1.4$ обобшают соответствуюшие результаты работы [87] на случай вырожденных матриц. Они позволяют взглянуть на некоторые известные результаты с новой точки зрения. Отправной точкой здесь является тот неожиданный факт, что любое замкнутое, выпуклое, центрально симметричное множество $E$ можно представить как пересечение эллипсоидов по меньшей мере тремя разными способами. 
ТеОРема 1.4.9. Пусть $E \subset \mathbb{R}^{n}$ будет выпуклым. замкнутым, иентрально симметричным множеством и $E^{0}$ будет его полярой. Определим матричные мно скества

$$
\mathscr{K}_{0}:=\left\{K=y y^{T}, y \in E^{0}\right\}, \mathscr{K}_{1}:=\operatorname{conv} \mathscr{K}_{0}, \mathscr{K}_{2}:=\{K \geqslant 0 \mid\langle K e, e\rangle \leqslant 1 \forall e \in E\} .
$$

Тогда $\mathscr{K}_{0} \subset \mathscr{K}_{1} \subset \mathscr{K}_{2}$ и любое множество $\mathscr{K}=\mathscr{K}_{i}$ подойдет для представления (4.16).

ДокАЗАТЕЛЬСтво. Напомним, что $E^{00}=E$, т.е.

$$
E=\left\{e \in \mathbb{R}^{n}:\langle e, y\rangle \leqslant 1 \forall y \in E^{0}\right\}
$$

Поскольку поляра $E^{0}$ центрально-симметрична, то

$$
E=\left\{e \in \mathbb{R}^{n}:\langle e, y\rangle^{2} \leqslant 1 \forall y \in E^{0}\right\}
$$

Это дает (4.16) для матричного множества $\mathscr{K}=\mathscr{K}_{0}$.

Очевидно, $\mathscr{K}_{0} \subset \mathscr{K}_{1}$. Покажем, что $\mathscr{K}_{1} \subset \mathscr{K}_{2}$. С этой целью рассмотрим любую матрицу $K=y y^{T}, y \in E^{0}$, принадлежащую множеству $\mathscr{K}_{0}$. Тогда $\langle K e, e\rangle=$ $\langle e, y\rangle^{2} \leqslant 1 \forall e \in E$. Поскольку $\langle K e, e\rangle=\langle e, y\rangle^{2} \geqslant 0$ для любого $e \in \mathbb{R}^{n}$, то $K \geqslant 0$. Поэтому $K \in \mathscr{K}_{2}$ для любой матрищы $K \in \mathscr{K}_{0}$, т.е. $\mathscr{K}_{0} \subset \mathscr{K}_{2}$. Множество $\mathscr{K}_{2}$ выпукло, поэтому оно содержит вьпуклые комбинации матрищ из множества $\mathscr{K}_{0} \subset \mathscr{K}_{2}$, так что $\mathscr{K}_{1}:=\operatorname{conv} \mathscr{K}_{0} \subset \mathscr{K}_{2}$.

Итак, $\mathscr{K}_{0} \subset \mathscr{K}_{1} \subset \mathscr{K}_{2}$ и (4.16) вьполнено с $\mathscr{K}=\mathscr{K}_{0}$. Легко понять, что остается проверить равенство (4.16) для матричного множества $\mathscr{K}_{2}$. Пусть $F$ обозначает правую часть (4.16) с $\mathscr{K}=\mathscr{K}_{2}$. Если $e \in E$, то $e \in F$ по определению множества $F$. Наоборот, пусть $e \in F$. Как было показано выше, для любого $y \in E^{0}$ матрица $K=y y^{T}$ принадлежит множеству $\mathscr{K}_{2}$. Поэтому $\langle K e, e\rangle=\langle e, y\rangle^{2} \leqslant 1 \forall y \in E^{0}$, и ввиду (4.25) мы имеем $e \in E$.

ЗАмЕчАнИЕ 1.4.10. Поляру $E^{0}$ можно заменить в теореме 1.4 .9 на множество всех ее крайних точек, если она ограниченна.

ДокАЗАТЕльство. Есливыпуклоемножество $C$ компактно, то $[45] C=\operatorname{conv} \operatorname{ext} C$, где ext $C$ обозначает множество всех крайних точек множества $C$. Поэтому можно переписать (4.24) как

$$
\begin{aligned}
E & =\left\{e \in \mathbb{R}^{n}: \delta^{*}\left(e \mid E^{0}\right) \leqslant 1\right\} \\
& =\left\{e \in \mathbb{R}^{n}: \delta^{*}\left(e \mid \operatorname{ext} E^{0}\right) \leqslant 1\right\} \\
& =\left\{e \in \mathbb{R}^{n}:\langle e, y\rangle^{2} \leqslant 1 \forall y \in \operatorname{ext} E^{0}\right\} .
\end{aligned}
$$

ЗАмечАнИЕ 1.4.11. Множество $\mathscr{K}_{2}$ дает все эллипсоиды $\langle K e, e\rangle \leqslant 1$, описанные вокруг множества $E$. 
С ЛЕДСТВИЕ 1.4.12. Пусть $\mathscr{K}$ будет любым из матричных множеств $\mathscr{K}_{0}, \mathscr{K}_{1}$, $\mathscr{K}_{2}$ или даже $\mathscr{K}_{3}:=\operatorname{conv}\left\{K=y y^{T} \mid y \in \operatorname{ext} E^{0}\right\}$, когда поляра $E^{0}$ ограниченна. Тогда справедлива формула (4.16a).

Случаи $\mathscr{K}=\mathscr{K}_{i}, i=1,2,3$, в чуть иной форме получены В. Б. Меласом [38]. При $\mathscr{K}=\mathscr{K}_{0}(4.16 \mathrm{a})$ преврашается в хорошо известное равенство [45]

$$
\delta^{*}(y \mid E)=\inf _{\mu>0}\left\{\mu \mid y \in \mu E^{0}\right\}
$$

Важньй вопрос - является ли сопряженная функция $D^{*}(y)$ всюду конечной? Если ответ отрицателен, то двойственная задача (2.2.3) из главы 2 будет содержать неявные ограничения. Согласно теореме 1.4.2 это не так, если множество $\mathscr{L}$, определенное в (4.9а), равно нулю. Очевидно, $\mathscr{L}=\{0\}$, если множество $\mathscr{K}$ содержит невырожденную матрищу. Следуюший результат показьвает, что когда множество $\mathscr{K}$ вьпукло, сушествование невырожденной матрищы $K \in \mathscr{K}$ необходимо и достаточно для того, чтобы сопряженная функция $D^{*}(y)$ была всюду конечной.

ТЕОрема 1.4.13. Пусть $\mathscr{K}$ будет выпуклым множеством неотричательно определенных матрии, для которых множество (4.9а) равно нулю. Тогда существует положстельно определенная матрица $K \in \mathscr{K}$.

ДоКАЗАТЕЛЬСТВо. В противном случае вьпуклое множество $\mathscr{K}$ и вьпукльй конус положительно определенных матриц не пересекаются. По теореме отделимости [2], [45] существует симметрическая матрица $A \neq 0$ такая, что

$$
\inf _{P>0}\langle A, P\rangle \geqslant \sup _{K \in \mathscr{K}}\langle A, K\rangle
$$

где

$$
\langle A, B\rangle:=\sum_{i=1}^{m} \sum_{j=1}^{n} a_{i j} b_{i j}=\operatorname{Sp} A B^{T}
$$

обозначает скалярное произведение прямоугольных матриц $A=\left\|a_{i j}\right\|$ и $B=\left\|b_{i j}\right\|$, $i=1, \ldots, m, j=1, \ldots, n$.

Как известно [9],

$$
\begin{gathered}
A \geqslant 0, B \geqslant 0,\langle A, B\rangle=0 \Longrightarrow A B=0 ; \\
A \geqslant 0 \Longleftrightarrow\langle A, B\rangle \geqslant 0 \forall B \geqslant 0 .
\end{gathered}
$$

Поскольку правая часть (4.28) конечна, то $A \geqslant 0$ и левая часть равна нулю. Поэтому $\langle A, K\rangle \leqslant 0 \forall K \in \mathscr{K}$, так что ввиду $(4.31)\langle A, K\rangle=0 \forall K \in \mathscr{K}$.

Согласно (4.30) это означает, что $A K=0 \forall K \in \mathscr{K}$. Поскольку $A \neq 0$, матрица $A$ имеет ненулевую строку $x$, и мы получаем из последнего равенства $K x=0 \forall k \in \mathscr{K}$, т.е. $0 \neq x \in \mathscr{L}$, противоречие. 


\section{§ 1.5. Двойственность невыпуклых экстремальных задач в случае гладкости двойственной задачи}

1.5.1. Рокафелларова схема двойственности. В этой схеме рассматривается функционал

$$
g(y):=\inf _{x \in X} \Phi(x, y)
$$

где $\Phi(x, y): X \times Y \rightarrow \overline{\mathbb{R}}-$ произвольная (невыпуклая) функция. Исходная задача заключается в минимизации (5.1) при $y=0$. В двойственной задаче нужно найти минимум

$$
g^{* *}(0)=-\inf _{y^{*} \in Y^{*}} g^{*}\left(y^{*}\right)
$$

где

$$
g^{*}\left(y^{*}\right)=\sup _{x \in X} \Phi_{x}^{*}\left(y^{*}\right), \quad \Phi_{x}(y):=\Phi(x, y) .
$$

Предположим, что маргинальная функция (5.1) (слабо) пн. снизу. Для этого достаточно, например, потребовать, что $X-$ компакт и функция $\Phi(x, y)$ пн. снизу на $X \times Y$ ( $Y$ снабжается слабой топологией, если используется производная Гато).

Теорема 1.5.1. Если функиия $g^{*}\left(y^{*}\right): Y^{*} \rightarrow \overline{\mathbb{R}}$ (Гато) Фреше-дифференцируема в точке своего минимума $\bar{y}^{*}$, то справедлива двойственность (1.2). Элемент $\bar{x} \in X$ тогда и только тогда дает глобальное решение задачи (5.1), когда $\Phi(\bar{x}, 0)+g^{*}\left(\bar{y}^{*}\right)=0[47]$.

1.5.2. Лагранжева схема двойственности. Пусть

$$
g(y)=\min _{x \in X}\left\{f_{0}(x) \mid F(x)+y=0\right\},
$$

где $Y$ - локально вьпуклое топологическое векторное пространство, функщия $f_{0}(x): X \rightarrow \mathbb{R}$ пн. снизу, а функция $F(x): X \rightarrow Y$ непрерьвна на компактном топологическом пространстве $X$.

ТеОРема 1.5.2. Если двойственная задача (5.2), әде

$$
g^{*}\left(y^{*}\right)=-\min _{x \in X} L\left(x, y^{*}\right), \quad L\left(x, y^{*}\right):=f_{0}(x)+\left\langle y^{*}, F(x)\right\rangle,
$$

имеет решение $\bar{y}^{*}$ такое, что абсолютный минимум (5.4) лагранжсиана $L\left(\cdot, \bar{y}^{*}\right)$ достигается на единственном әлементе $\bar{x} \in X$, то справедлива двойственность (1.2). В этом случае әлемент $\bar{x}$ дает абсолютное решение исходной задачи (5.3) для $y=0[47]$.

ЗАмечАниЕ. Когда исходная задача содержит также ограничения типа неравенств

$$
f_{i}(x)+y_{i} \leqslant 0, \quad i=1, \ldots, k,
$$

где $f_{i}(x): X \rightarrow \mathbb{R}$ пн. снизу функции, сопряженная функция $g^{*}\left(y^{*}\right)$ равняется (5.4), если $y_{i}^{*} \geqslant 0, i=1, \ldots, k$; иначе она бесконечна. Тем не менее, теорема 1.5.2 остается справедлива в той же самой формулировке [87].

Для квадратичных задач аналогичные результатыполучены Н. З. Шором [63], [64]. 
1.5.3. ПримеРы. 1) В некоторых задачах автоматического управления и теории операторных пучков [1], [59] встречается задача $(5.3),(5.5)$ в случае $f_{i}(x)=\left\langle C_{i} x, x\right\rangle$, $i=0,1, \ldots, m, A=\left\{x \in \mathbb{R}^{n},|x|=1\right\}$. Двойственная задача (5.2) состоит в максимизации минимального собственного числа матричного пучка

$$
C\left(y^{*}\right):=C_{0}+y_{1}^{*} C_{1}+\cdots+y_{m}^{*} C_{m}
$$

по переменньм $y^{*} \in \mathbb{R}^{m}, y_{i}^{*} \geqslant 0, i=1, \ldots, k$. Если эта задача имеет решение $y^{*}=\bar{y}^{*}$, причем минимальное собственное число матрицы $C\left(\bar{y}^{*}\right)$ является простым, то согласно теореме 1.5.2 соответствующий ему нормированный собственный вектор дает глобальное решение исходной задачи (5.3), (5.5).

Любопытно, что недавно этот пример встретился также в теории оптимального планирования эксперимента [85].

2) Пусть в задаче $(5.3),(5.5) f_{i}(x)=\left\langle C_{i} x, x\right\rangle / 2-\left\langle a_{i}, x\right\rangle-b_{i}, i=0,1, \ldots, m, A=\mathbb{R}^{n}$. Тогда сопряженная функция (5.4) конечна лишь для тех векторов $y^{*}$, для которых оператор (5.6) неотрищательно определен и его образ содержит вектор $a\left(y^{*}\right):=a_{0}+$ $y_{1}^{*} a_{1}+\cdots+y_{m}^{*} a_{m}$. Теорема 1.5.2 с последуюшим замечанием дают результат, полученньй в работе [63]: если решение $y^{*}=\bar{y}^{*}$ двойственной задачи (5.2) удовлетворяет условиям $\bar{y}_{i}^{*}>0, i=1, \ldots, k, C\left(\bar{y}^{*}\right)>0$, то $\bar{x}=C^{-1}\left(\bar{y}^{*}\right) a\left(\bar{y}^{*}\right)$ - глобальное решение исходной задачи $(5.3),(5.5)$.

Из многочисленных работ, посвяшенных теории двойственности, отметим [1], [2], $[4],[19],[21],[26],[39],[42]-[47],[59],[64],[65],[69],[76],[77],[87],[96],[100]$.

\section{Глава 2}

\section{Минимаксное оценивание параметров \\ в линейных моделях наблюдения с неопределенными статистиками второго порядка}

\section{§.1. Постановка задачи}

Рассмотрим линейную модель

$$
\eta=A \theta+\xi, \quad l=C^{T} \theta,
$$

в которой $\eta \in \mathbb{R}^{N}$ - вектор измерений, $\theta \in \mathbb{R}^{m}$ - вектор неизвестных параметров, $l \in \mathbb{R}^{r}$ - оцениваемьй вектор, $\xi$ - вектор случайных ошибок, имеющий среднее $\mathbb{E} \xi$ и ковариационную матрицу $K:=\operatorname{cov}(\xi, \xi)=\mathbb{E}(\xi-\mathbb{E} \xi)(\xi-\mathbb{E} \xi)^{T}$, которые принадлежат априорно известным множеству $\mathscr{E} \subset \mathbb{R}^{N}$ и множеству $\mathscr{K}$ положительно определенных матриц порядка $N$ :

$$
\mathbb{E} \xi \in \mathscr{E}_{0}, \quad K \in \mathscr{K}
$$

Предполагается, что множество $\mathscr{K}$ ковариационных матриц $K$ имеет вид

$$
\mathscr{K}=\left\{K=D+\sum_{i=1}^{k} e_{i} e_{i}^{T} \mid D \in \mathscr{D}, e_{i} \in \mathscr{E}_{i}, i=1, \ldots, k\right\}
$$


(см. [5]), который возникает, например, когда суммарная ошибка $\xi$ может быть разбита на сумму $\xi=\xi_{0}+\varepsilon_{1} e_{1}+\cdots+\varepsilon_{k} e_{k}$ некоррелированных слагаемых $\xi_{0}, \varepsilon_{1} e_{1}, \ldots, \varepsilon_{k} e_{k}$, если векторы $e_{i} \in \mathscr{E}_{i}, i=1, \ldots, k$, не случайны, и выполнены условия $\mathbb{E} \xi_{0} \in \mathscr{E}_{0}$, $\operatorname{cov}\left(\xi_{0}, \xi_{0}\right) \in \mathscr{D}, \mathbb{E} \varepsilon_{i}=0, \mathbb{E} \varepsilon_{i}^{2}=1, i=1, \ldots, k$ (здесь $\xi_{0}$ - ошибка общей природы, а $\varepsilon=\left(\varepsilon_{1}, \ldots, \varepsilon_{k}\right)$ - стандартньй белый шум). Будем считать, что все множества $\mathscr{E}_{i} \subset \mathbb{R}^{N}$ и $\mathscr{D}$ выпуклы, замкнуты и ограниченны, причем множества $\mathscr{E}_{i}, i=0, \ldots, k$, центрально-симметричны, и что матрица $A$ имеет полньй ранг $m \leqslant N$.

Сначала мы будем рассматривать линейные оценки $\hat{l}=\left(\hat{l}_{1}, \ldots, \hat{l}_{r}\right), \hat{l}_{j}=\left\langle\eta, x_{j}\right\rangle$, $x_{j} \in \mathbb{R}^{N}, j=1, \ldots, r$, заданного параметра $l=C^{T} \theta \in \mathbb{R}^{r}$, которые имеют ошибки $\delta l_{j}=\hat{l}_{j}-l_{j}=\left\langle A^{T} x_{j}-c_{j}, \theta\right\rangle+\left\langle\xi, x_{j}\right\rangle, j=1, \ldots, r$, где через $c_{j} \in \mathbb{R}^{m}$ обозначены векторы-столбцы матрицы $C=\left(c_{1}, \ldots, c_{r}\right) \in \mathbb{R}^{m r}$. Максимальное (гарантированное) значение

$$
2 D(X):=\sup _{\theta, \xi} \mathbb{E}|\delta l|^{2}, \quad X:=\left(x_{1}, \ldots, x_{r}\right) \in \mathbb{R}^{N r},
$$

среднеквадратичной ошибки $\mathbb{E}|\delta l|^{2}$ будет конечным при условии $A^{T} X=C$. В этом случае

$$
2 D(X)=\max _{B \in \mathscr{B}} \sum_{j=1}^{r}\left\langle B x_{j}, x_{j}\right\rangle=\max _{B \in \mathscr{B}}\langle B X, X\rangle,
$$

где $\mathscr{B}$ - априорное множество матриц вторых моментов вектора $\xi$

$$
\mathscr{B}=\left\{B=D+\sum_{i=0}^{k} e_{i} e_{i}^{T} \mid D \in \mathscr{D}, e_{i} \in \mathscr{E}_{i}, i=0, \ldots, k\right\}
$$

(в дальнейшем удобно использовать для всех переменных матричные обозначения. Будем считать, что матрицы $X, Y \in \mathbb{R}^{N r}$, имеющие по $N$ строк и $r$ столбцов, лежат в конечномерном евклидовом пространстве $\mathbb{R}^{N r}$ со скалярным произведением (1.4.29)). Чтобы найти минимаксную оценку $\hat{l}_{*}=X_{*}^{T} \eta$, нужно решить задачу

$$
\delta_{\min }^{2} / 2:=\min \left\{D(X): X \in \mathbb{R}^{N r}, A^{T} X=C\right\} .
$$

\section{$\S 2.2$. Теорема двойственности}

$\mathrm{B} \S \S 2.2,2.3$ мы будем предполагать, что либо оценивается скалярньй параметр $l$, либо ошибки наблюдений не содержат систематических составляюших $\varepsilon_{i} e_{i}$.

Лемма 2.2.1. Пусть либо $l \in \mathbb{R}$, либо множество $\mathscr{B}=\mathscr{D}$ компактно и выпукло. Тогда сопряженная функция $D^{*}(Y)$ непрерывно дифференцируема и может быть записана в форме

$$
D^{*}(Y)=\min _{B \in \mathscr{B}}\left\langle B^{-1} Y, Y\right\rangle / 2 .
$$

Для фиксированного вектора $Y=\left(y_{1}, \ldots, y_{r}\right) \in \mathbb{R}^{N r}$ матрица $B_{Y} \in \mathscr{B}$ тогда и только тогда дает решение вспомогательной задачи (2.1), когда она дает решение задачи (1.5) для вектора $X_{Y}=B_{Y}^{-1} Y$. В этом случае

$$
D^{*}(Y)=D\left(X_{Y}\right), \quad \nabla D^{*}(Y)=X_{Y}=B_{Y}^{-1} Y .
$$

Доказательство следует из теорем 1.4.2 и 1.4.3.

Помимо матрицы $C=\left(c_{1}, \ldots, c_{r}\right) \in \mathbb{R}^{m r}$ мы будем также рассматривать матрищы $\Theta=\left(\theta_{1}, \ldots, \theta_{r}\right) \in \mathbb{R}^{m r}$, где $\theta_{j} \in \mathbb{R}^{m}, j=1, \ldots, m$. 
Теорема 2.2.2. Справедливо равенство

$$
-\sigma_{\min }^{2} / 2=\min _{\Theta \in \mathbb{R}^{m r}}\left\{D^{*}(A \Theta)-\langle C, \Theta\rangle\right\} .
$$

Если $\Theta_{*}-$ решение двойственной задачи (2.3), то вектор

$$
X_{*}=\nabla D^{*}\left(A \Theta_{*}\right)
$$

является единственным решением исходной задачи (1.5)-(1.7) [88].

Доказательство следует из стандартной теоремы двойственности вьпуклого программирования [2], [42].

Таким образом, вместо исходной негладкой задачи (1.7), в которой может быть очень много переменных, удобно перейти к двойственной задаче $(2.3)$, в которой сопряженная функция $D^{*}(Y): \mathbb{R}^{N r} \rightarrow \mathbb{R}$ непрерьвно дифференцируема и для ряда практически важных случаев статистических ограничений вычислена аналитически (см. [48], [50]). В этих случаях двойственная задача (2.3) заключается в безусловной минимизации эффективно вычисляемой гладкой вьпуклой функции вектора состояния, имеюшей небольшую размерность (обычно $m \ll N$ ). В [50], [52], [88] описаны несколько алгоритмов построения итерационных последовательностей $\left\{\Theta_{s}\right\}, s=0,1, \ldots$, дающих решение двойственной задачи (2.3).

\section{§ 2.3. Минимаксные оценки и метод наименьших квадратов}

TеОРема 2.3.1. Пусть либо $l \in \mathbb{R}$, либо множество $\mathscr{B}=\mathscr{D}$ компактно и выпукло и $\hat{l}_{*}=\mathscr{X}_{*}^{T} \eta$, əде

$$
\mathscr{X}_{*}=B_{*}^{-1} A\left(A^{T} B_{*}^{-1} A\right)^{-1} C
$$

- МНК-оцениватель, соответствующий весовой матрице $B_{*} \in \mathscr{B}$ и имеющий среднеквадратичную ошибку

$$
\mathbb{E}\left|\delta l_{*}\right|^{2}=\max _{B \in \mathscr{B}}\left\langle\left(A^{T} B^{-1} A\right)^{-1} C, C\right\rangle .
$$

Тогда $\sigma_{\min }^{2}=\mathbb{E}\left|\delta l_{*}\right|^{2}$, очениватель $\mathscr{X}_{*}$ является решением минимаксной задачи (1.5)-(1.7), а вектор

$$
\Theta_{*}=\left(A^{T} B_{*}^{-1} A\right)^{-1} C
$$

является решением двойственной задачи (2.3). И наоборот, если вектор $\Theta_{*}$ - решение двойственной задачи (2.3), а матрица $B_{*}$ - решение вспомогательной задачи (2.1), вычисленное для вектора $Y=A \Theta_{*}$, то $B_{*}-$ наименее благоприятная матрица вторых моментов (решение задачи (3.2)) и справедливь равенства (3.1), (3.3) [88].

Доказательство вытекает из теоремы двойственности (2.3) и равенства (2.1) (см. [88]).

Эта теорема является обобщением теоремы Гаусса-Маркова (по-видимому, связь минимаксных оценок с методом наименьших квадратов впервые была замечена еще Вальдом [90]; см. также [70], [78], [81], [84]). Для случая, когда $\mathscr{E}_{j}=\{0\}, j=0, \ldots, r$, близкие задачи рассматривались И. Ф. Пинелисом [41]. 


\section{§.4. Линейность минимаксных оценок}

Как известно, в задачах минимаксного оценивания можно ограничиться линейными алгоритмами, если ошибки измерений не случайны (см. работы Н.С. Бахвалова [6], А.Б. Куржанского [26] и других [12], [13], [34]-[36], [79], [80], [83], [99]). Здесь этот результат устанавливается для скалярного оцениваемого параметра $l$ и случайных ошибок измерений, имеюших ковариационную матрицу из априорного класса (1.3).

Введем обозначение $\Xi=\left\{\xi: \mathbb{E} \xi=0, \mathbb{E} \xi \xi^{T} \in \mathscr{B}\right\}$. Для любой нелинейной оценки $\hat{l}(\eta)$ параметра $l$ гарантированное значение среднеквадратичного отклонения $\mathbb{E}\left(\delta l^{2}\right)$ ошибки $\delta l=\hat{l}-l$ равно

$$
D(\hat{l}):=\sup \left\{\mathbb{E}\left(\delta l^{2}\right) \mid \eta=A \theta+\xi, \xi \in \Xi, \theta \in \mathbb{R}^{m}\right\},
$$

и задача отыскания оптимального алгоритма минимаксного оценивания в классе нелинейных оценок принимает вид

$$
D_{\min }:=\inf _{\hat{l}(\cdot)} D(\hat{l}) .
$$

В дальнейшем в (4.2) будут рассматриваться только те оценки $\hat{l}(\eta)$, для которых выполнены условия регулярности (см. [16, с. 252]) в неравенстве Рао-Крамера для всех нормальных распределений $\eta \sim \mathscr{N}(A \theta, B), B \in \mathscr{B}$.

Teopema 2.4.1. Пусть $l=c^{T} \theta \in \mathbb{R}, \xi \in \Xi u \mathscr{E}_{0}=\{0\}$. Тогда

$$
D_{\min }=\max _{B \in \mathscr{B}} c^{T}\left(A^{T} B^{-1} A\right)^{-1} c .
$$

Решение задачи (4.1)-(4.2) достигается на линейной оченке

$$
\hat{l}_{*}=c^{T}\left(A^{T} B_{*}^{-1} A\right)^{-1} A^{T} B_{*}^{-1} \eta,
$$

соответствующей обобщенному методу наименьших квадратов с весовой матрицей $B_{*} \in \mathscr{B}$, которая дает решение задачи (4.3). При этом наименее благоприятно нормальное распределение $\xi \sim \mathscr{N}\left(0, B_{*}\right)[51]$.

В [7], [88] рассматривался класс $\mathscr{P}_{1}$ всех распределений вектора $\xi$, удовлетворяющих ограничениям (1.2). В классе линейных оценок минимаксная оценка зависит только от матрицы вторых моментов $B=\mathbb{E}_{P} \xi \xi^{T}$, которая принадлежит множеству (1.6). Оказьвается, что если ввести более широкий класс $\mathscr{P}_{2}$ всех распределений $P(x)$, удовлетворяюших ограничению $\mathbb{E}_{P} \xi \xi^{T} \in \mathscr{B}$ с априорно заданным множеством $\mathscr{B}$ вида (1.6) (распределения из классов $\mathscr{P}_{1}$ и $\mathscr{P}_{2}$ имеют одни и те же матрищы вторых моментов), то минимаксная оценка тоже будет линейной. 
Teopema 2.4.2. Пусть $l=c^{T} \theta \in \mathbb{R} u \xi \in \Xi=\left\{\xi: \mathbb{E} \xi \xi^{T} \in \mathscr{B}\right\}$. Тогда справедливо равенство (4.3). Решение задачи (4.1)-(4.2) достигается на линейной оченке (4.4) с весовой матрицей $B_{*} \in \mathscr{B}$, которая дает решение задачи (4.3). При этом наименее благоприятныцм является нормальное распределение $\xi \sim \mathscr{N}\left(0, B_{*}\right)$.

Доказательства теорем 2.4.1, 2.4.2 опираются на неравенство Рао-Крамера и теорему 2.3.1 (см. [51], где рассмотрен случай вьпуклого множества $\mathscr{B}=\mathscr{D}$ ). Для задачи о наихудшей корреляции линейность минимаксной оценки установлена А. И. Матасовым [37]. В стохастических постановках фундаментальную роль играет теорема о нормальной корреляции [33], [53], [89].

Для априорного класса $\mathscr{P}_{1}$ вопрос о линейности оценок остается открытым (нужно заметить, что в близких задачах ответ отрицателен [72], [75]). Дальнейшая литература к главе 2: [3], [7], [8], [10], [11], [14], [17], [18], [20], [22]-[28], [30]-[33], [40], [41], [52], $[60],[61],[70],[71],[74],[78],[84],[86]-[90]$.

\section{Глава 3.}

\section{Приложения к теории плохо обусловленных задач}

\section{$\S 3.1$. Постановка задачи}

Теперь мы будем рассматривать задачу восстановления параметра $l=\langle c, \theta\rangle$ из вектора данных $\eta=A \theta+\xi$. Эта задача плохо обусловлена, если отношение наибольшего к наименьшему сингулярному числу матришы $A$ велико. В этом случае малые ошибки в векторе данных $\eta$ могут привести к большим ошибкам в векторе регрессионных коэффициентов $\theta$. Обший способ уменьшения этих ошибок заключается в использовании априорной информации $\theta \in \Theta$.

Предполагается, что вектор случайных ошибок $\xi \in \mathbb{R}^{N}$ удовлетворяет априорньм ограничениям $\mathbb{E} \xi=0, K=\mathbb{E} \xi \xi^{T} \in \mathscr{K}$, где $\mathscr{K}$ - это матричное множество (2.1.3), состоящее из положительно определенных матрищ порядка $N$. Здесь и далее мы будем рассматривать линейные оценки $\hat{l}=\langle x, \eta\rangle+t, x \in \mathbb{R}^{N}, t \in \mathbb{R}$, заданного скалярного параметра $l=\langle c, \theta\rangle, c \in \mathbb{R}^{m}$, которые имеют ошибку $\delta l:=\hat{l}-l=\langle x, \xi\rangle+$ $\left\langle A^{T} x-c, \theta\right\rangle+t$. Максимальное (гарантированное) значение (2.1.4) среднеквадратичной ошибки $\mathbb{E} \delta l^{2}$ равно

$$
d(\hat{l})=\max _{K \in \mathscr{K}}\langle K x, x\rangle+\max _{\theta \in \Theta}\left(\left\langle A^{T} x-c, \theta\right\rangle+t\right)^{2} .
$$

Чтобы найти минимаксную оценку $\hat{l}_{*}=\left\langle x_{*}, \eta\right\rangle+t_{*}$, нужно решить задачу

$$
D_{\min }=\min _{\hat{l}=\langle x, \eta\rangle+t} d(\hat{l}) .
$$

Стоит отметить, что регрессионная матрица $A$ произвольна. Вполне может быть, что $N<m$, но даже если $N \geqslant m$, информационная матрица $A^{T} K^{-1} A$ может быть вырожденной. Случай квадратных регрессионных матриц встречается при решении 
линейных алгебраических систем $A \theta=y$. Они возникают также после дискретизации линейных интегральных уравнений, так что результаты главы 3 можно использовать в весьма разных областях (см. [58]).

Сушествует обширная теория плохо обусловленных задач в гильбертовых и даже банаховых пространствах, включающая рассмотрение задач оценивания скалярных и векторных параметров в терминах минимакса (наиболее близкими по постановкам и методам работами являются [29], [58]). Но, как отмечается в работах А. М. Федотова [58] и И. А. Ибрагимова и Р.З. Хасьминского [17], [18], им не удалось доказать соответствуюшие результаты, используя стандартные теоремы о минимаксе. В нашей работе используются теоремы о минимаксе, доказанные в главе 1.

\section{$\S$ 3.2. Теорема двойственности}

Сначала мы сосредоточимся на случае центрально-симметричного множества $\Theta$. Все множества $\mathscr{D}, \mathscr{E}_{i}$ в (2.1.3), а также множество $\Theta$ предполагаются вьпукльми и компактньми, причем множества $\mathscr{E}_{i}$ симметричны относительно нуля.

Теорема 3.2.1. Справедлива двойственность

$$
D_{\min }=\max _{K \in \mathscr{K}, \theta \in \Theta} \frac{\langle c, \theta\rangle^{2}}{1+\left\langle K^{-1} A \theta, A \theta\right\rangle} .
$$

Если $K_{*} \in \mathscr{K}, \theta_{*} \in \Theta$ - решение двойственной задачи (2.1), то

$$
\hat{l}_{*}=\frac{\left\langle c, \theta_{*}\right\rangle}{1+\left\langle K_{*}^{-1} A \theta_{*}, A \theta_{*}\right\rangle}\left\langle K_{*}^{-1} A \theta_{*}, \eta\right\rangle
$$

- это решение исходной задачи (1.1)-(1.2).

ДокАЗАТЕльство. Определим вектор $x_{0}:=c-A^{T} x \in \mathbb{R}^{m}$. При фиксированных $x \in \mathbb{R}^{N}$ минимум функции (1.1) по переменной $t \in \mathbb{R}$ достигается при $t_{*}=0$. Действительно, поскольку множество $\Theta$ симметрично относительно нуля и

$$
\left\langle x_{0}, \theta\right\rangle^{2} \leqslant \max \left[\left(t-\left\langle x_{0}, \theta\right\rangle\right)^{2},\left(t+\left\langle x_{0}, \theta\right\rangle\right)^{2}\right]
$$

то

$$
\max _{\theta \in \Theta}\left\langle x_{0}, \theta\right\rangle^{2} \leqslant \max _{\theta \in \Theta}\left(t-\left\langle x_{0}, \theta\right\rangle\right)^{2}
$$

для всех $t \in \mathbb{R}$. Поэтому исходная задача (1.2) принимает вид

$$
D_{\min } / 2=\min _{x, x_{0}}\left\{D(x)+D_{0}\left(x_{0}\right) \mid A^{T} x+x_{0}=c\right\},
$$

где

$$
2 D(x):=\max _{K \in \mathscr{K}}\langle K x, x\rangle, \quad 2 D_{0}\left(x_{0}\right):=\max _{\theta \in \Theta}\left\langle x_{0}, \theta\right\rangle^{2}=\delta^{* 2}\left(x_{0} \mid \Theta\right) .
$$

Теперь можно воспользоваться теоремой 2.2.2:

$$
D_{\min } / 2=\max _{q \in \mathbb{R}^{m}}\left\{\langle c, q\rangle-D^{*}(A q)-D_{0}^{*}(q)\right\},
$$


где согласно теореме 1.4.2 и следствию 1.4 .12

$$
\begin{aligned}
& 2 D_{0}^{*}(q)=\min _{\theta \in \Theta}\left\langle\left(\theta \theta^{T}\right)^{+} q, q\right\rangle, \\
& 2 D^{*}(y)=\min _{K \in \mathscr{K}}\left\langle K^{-1} y, y\right\rangle .
\end{aligned}
$$

Поскольку $\left(\theta \theta^{T}\right)^{+}=\theta \theta^{T} /\langle\theta, \theta\rangle^{2}$, а образ матрищы $\theta \theta^{T}$ состоит из векторов $q=\lambda \theta$, $\lambda \in \mathbb{R}$, то

$$
\begin{aligned}
D_{\min } / 2 & =\max _{q=\lambda \theta} \max _{K \in \mathscr{K}, \theta \in \Theta}\left\{\langle c, q\rangle-\left\langle K^{-1} A q, A q\right\rangle / 2-\langle\theta, q\rangle^{2} /\left(2\langle\theta, \theta\rangle^{2}\right)\right\} \\
& =\max _{K \in \mathscr{K}, \theta \in \Theta} \max _{\lambda \in \mathbb{R}}\left\{\lambda\langle c, \theta\rangle-\lambda^{2}\left\langle K^{-1} A \theta, A \theta\right\rangle / 2-\lambda^{2} / 2\right\} .
\end{aligned}
$$

При фиксированных $K \in \mathscr{K}$ и $\theta \in \Theta$ последний максимум достигается при $\lambda=$ $\frac{\langle c, \theta\rangle}{1+\left\langle K^{-1} A \theta, A \theta\right\rangle}$, что дает (2.1).

Если $K_{*} \in \mathscr{K}, \theta_{*} \in \Theta$ - решение двойственной задачи (2.1), то из доказанного следует, что вектор

$$
q_{*}=\lambda_{*} \theta_{*}=\frac{\left\langle c, \theta_{*}\right\rangle}{1+\left\langle K_{*}^{-1} A \theta_{*}, A \theta_{*}\right\rangle} \theta_{*}
$$

дает решение задачи (2.4). Тогда, как и в теореме 2.2.2, векторы

$$
x_{*}=\nabla D^{*}\left(A q_{*}\right)=K_{*}^{-1} A q_{*}, \quad x_{0}^{*}=c-A^{T} x_{*}
$$

являются решением исходной задачи (2.3). Ввиду равенства (2.7) это дает (2.2).

Теорема 3.2.1 является обобщением одного важного результата, принадлежащего И.А. Ибрагимову и Р.З. Хасьминскому [17], [18], а также А. М. Федотову [58], на задачи минимаксного оценивания с неопределенными статистиками второго порядка. Она обеспечивает многие возможности для конструирования численных алгоритмов, которые будут обсуждены в другой работе.

\section{§3.3. Оценка Кукса-Ольмана}

3.3.1. Неопределенные статистики второго порядка. Особо интересен случай, когда $\Theta$ - это эллипсоид

$$
\left\{\theta \in \mathbb{R}^{m} \mid\left\langle T^{-1} \theta, \theta\right\rangle \leqslant 1\right\} .
$$

Кукс и Ольман показали [23]-[25], что в этом случае минимаксной будет оценка $\hat{l}_{*}=\left\langle c, \hat{\theta}_{*}\right\rangle$, в которой вектор параметров

$$
\hat{\theta}_{*}=\left(A^{T} K^{-1} A+T^{-1}\right)^{-1} A^{T} K^{-1} \eta
$$

не зависит от оцениваемого скалярного параметра $l=\langle c, \theta\rangle$. Другое замечательное свойство оценки (3.2) состоит в том, что она совпадает с байесовской оценкой. 
Неожиданный результат, принадлежаший В. Б. Меласу [38], состоит в том, что и для произвольного выпуклого, центрально симметричного тела $\Theta$ минимаксная оценка является оценкой Кукса-Ольмана, связанной с некоторым эллипсоидом (3.1), описанным вокруг множества $\Theta$. Помимо рассмотрения неопределенных статистик случайных ошибок мы обобшаем этот результат в двух направлениях. Сначала мы будем рассматривать априорное множество

$$
\Theta=\bigcup_{T \in \mathscr{T}}\left\{\theta \in \mathbb{R}^{m}:\left\langle T^{-1} \theta, \theta\right\rangle \leqslant 1\right\}
$$

которое является объединением всех эллипсоидов $(3.1)$, когда $T$ пробегает матричное множество

$$
\mathscr{T}:=\left\{T=T_{0}+\sum_{i=1}^{k_{0}} c_{i} c_{i}^{T}: T_{0} \in \mathscr{T}_{0}, c_{i} \in C_{i}, i=1, \ldots, k_{0}\right\}
$$

Затем мы примем в качестве априорного множества пересечение

$$
\Theta=\bigcap_{T \in \mathscr{T}}\left\{\theta \in \mathbb{R}^{m}:\langle T \theta, \theta\rangle \leqslant 1\right\}
$$

всех эллипсоидов $\langle T \theta, \theta\rangle \leqslant 1$ с матрицами $T$, пробегаюшими множество (3.4). Мы покажем, что в обоих случаях минимаксная оценка может рассматриваться как оценка Кукса-Ольмана, связанная с некоторыми матрицами $K \in \mathscr{K}$ и $T \in \mathscr{T}$.

Отсюда следует, что минимаксная оценка всегда является оценкой Кукса-Ольмана, поскольку, как было показано в теореме 1.4.9, любое замкнутое, выпуклое, центрально симметричное тело $\Theta$ можно представить в виде (3.5) по меньшей мере тремя различными способами.

Здесь и далее мы будем предполагать, что все множества $\mathscr{T}_{0}, C_{i}$ в $(3.4)$ вьпуклы и компактны, множества $C_{i}$ симметричны относительно нуля и все матрицы $T_{0} \in \mathscr{T}_{0}$ неотрицательно определены.

ТЕОРема 3.3.1. Пусть априорное множество $\Theta$ имеет вид (3.3), а все матрицы Т в множсестве (3.4) невырожденны. Тогда справедлива двойственность

$$
D_{\min }=\max _{K \in \mathscr{K}, T \in \mathscr{T}}\left\langle\left(A^{T} K^{-1} A+T^{-1}\right)^{-1} c, c\right\rangle .
$$

Если $K_{*} \in \mathscr{K}, T_{*} \in \mathscr{T}-$ решение задачи (3.6), то оченка Кукса-Ольмана

$$
\hat{l}_{*}=\left\langle c, \hat{\theta}_{*}\right\rangle, \quad \hat{\theta}_{*}=\left(A^{T} K_{*}^{-1} A+T_{*}^{-1}\right)^{-1} A^{T} K_{*}^{-1} \eta,
$$

является решением исходной задачи (1.1)-(1.2). 
ДоКАЗАТЕЛЬСТво. По следствию 1.4.5 априорное множество (3.3) центрально симметрично, компактно и вьпукло. После замены переменных

$$
\theta^{\prime}=\theta /\left(1+\left\langle K^{-1} A \theta, A \theta\right\rangle\right)^{1 / 2}, \quad \theta=\theta^{\prime} /\left(1-\left\langle K^{-1} A \theta^{\prime}, A \theta^{\prime}\right\rangle\right)^{1 / 2}
$$

теорема двойственности (2.1) становится

$$
D_{\min }=\max _{K \in \mathscr{K}} \max _{\theta^{\prime} \in \Theta^{\prime}}\left\langle c, \theta^{\prime}\right\rangle^{2}
$$

где $\Theta^{\prime}$ - это объединение эллипсоидов

$$
E(T):=\left\{\theta^{\prime} \in \mathbb{R}^{m}:\left\langle\left(A^{T} K^{-1} A+T^{-1}\right) \theta^{\prime}, \theta^{\prime}\right\rangle \leqslant 1\right\}, \quad T \in \mathscr{T} .
$$

Если $K_{*} \in \mathscr{K}, \theta_{*}^{\prime} \in \Theta^{\prime}-$ решение двойственной задачи $(3.9)$, то согласно (2.2) оценка

$$
\hat{l}_{*}=\left\langle c, \theta_{*}^{\prime}\right\rangle\left\langle K_{*}^{-1} A \theta_{*}^{\prime}, \eta\right\rangle
$$

является решением исходной задачи (1.1)-(1.2). Теперь мы имеем

$$
\max _{\theta^{\prime} \in \Theta^{\prime}}\left\langle c, \theta^{\prime}\right\rangle^{2}=\max _{T \in \mathscr{T}} \max _{\theta^{\prime} \in E(T)}\left\langle c, \theta^{\prime}\right\rangle^{2}=\max _{T \in \mathscr{T}}\left\langle\left(A^{T} K^{-1} A+T^{-1}\right)^{-1} c, c\right\rangle,
$$

так что равенство (3.9) сводится к (3.6). Если $K_{*} \in \mathscr{K}, T_{*} \in \mathscr{T}$ - это решение задачи $(3.6)$, то пара $K_{*}, \theta_{*}^{\prime}$ с вектором

$$
\theta_{*}^{\prime}=\left(A^{T} K_{*}^{-1} A+T_{*}^{-1}\right)^{-1} c /\left\langle\left(A^{T} K_{*}^{-1} A+T_{*}^{-1}\right)^{-1} c, c\right\rangle^{1 / 2}
$$

является решением задачи (3.9), а оценка (3.10) принимает вид (3.7).

ТЕорема 3.3.2. Пусть априорное множество $\Theta$ компактно и имеет вид (3.5). Тогда

$$
D_{\min }=\max _{K \in \mathscr{K}} \min _{T \in \mathscr{T}}\left\langle\left(A^{T} K^{-1} A+T\right)^{+} c, c\right\rangle .
$$

Если $K_{*} \in \mathscr{K}, T_{*} \in \mathscr{T}$ - решение задачи (3.12), то оченка Кукса-Ольмана

$$
\hat{l}_{*}=\left\langle K_{*}^{-1} A\left(A^{T} K_{*}^{-1} A+T_{*}^{-1}\right)^{+} c, \eta\right\rangle
$$

является решением исходной задачи (1.1)-(1.2). 
ДокАЗАТЕльство. Опять мы используем замену переменных (3.8), после которой множество (3.5) заменяется на пересечение $\Theta^{\prime}$ эллипсоидов $E(T):=\left\{\theta^{\prime} \in \mathbb{R}^{m}\right.$ : $\left.\left\langle\left(A^{T} K^{-1} A+T\right) \theta^{\prime}, \theta^{\prime}\right\rangle \leqslant 1\right\}, T \in \mathscr{T}$. Поскольку множество $\Theta^{\prime}$ центрально симметрично, то по следствию 1.4.6 для фиксированной матрицы $K \in \mathscr{K}$ имеем

$$
\left.\max _{\theta^{\prime} \in \Theta^{\prime}}\left\langle c, \theta^{\prime}\right\rangle^{2}=\delta^{* 2}\left(c \mid \Theta^{\prime}\right)=\min _{T \in \mathscr{T}}\left(A^{T} K^{-1} A+T\right)^{+} c, c\right\rangle .
$$

Используя (3.9) и последнее равенство, получим (3.12).

Чтобы доказать (3.13), мы воспользуемся равенством (3.10), в котором $K_{*} \in \mathscr{K}$, $\theta_{*}^{\prime} \in \Theta^{\prime}$ - это решение двойственной задачи (3.9). В этот раз получаем, что пара $K_{*}, \theta_{*}^{\prime}$ с вектором

$$
\theta_{*}^{\prime}=\left(A^{T} K_{*}^{-1} A+T_{*}\right)^{+} c /\left\langle\left(A^{T} K_{*}^{-1} A+T_{*}\right)^{+} c, c\right\rangle^{1 / 2}
$$

является искомым решением, ибо в силу (3.12) в этом случае

$$
D_{\min }=\left\langle\left(A^{T} K_{*}^{-1} A+T_{*}\right)^{+} c, c\right\rangle=\left\langle c, \theta_{*}^{\prime}\right\rangle^{2},
$$

и поэтому равенство (3.10) преобразуется к виду (3.13).

3.3.2. Определенные статистики второго порядка. Теперь мы можем перейти к регрессионной модели, в которой вектор ошибок $\xi$ имеет точно определенные статистики первого и второго порядка, а вектор коэффициентов регрессии $\theta$ принадлежит произвольному вьпуклому центрально-симметричному телу $\Theta$. Хотя соответствующие минимаксные задачи были разобраны В. Б. Меласом [38] и А.М. Федотовым [58], по-видимому, некоторые результаты ускользнули от их внимания (во всяком случае, новьми являются представленные здесь доказательства).

СлЕДСТВИЕ 3.3.3. Пусть множество $\mathscr{K}=\{K\}$ будет одноточечным $и$

$$
\mathscr{T}=\mathscr{T}_{2}:=\{T \geqslant 0:\langle T \theta, \theta\rangle \leqslant 1 \forall \theta \in \Theta\}
$$

будет множеством всех эллипсоидов, описанных вокруг произвольного выпуклого, иентрально-симметричного тела $\Theta$. Тогда

$$
D_{\min }=\min _{T \in \mathscr{T}}\left\langle\left(A^{T} K^{-1} A+T\right)^{+} c, c\right\rangle
$$

Если $T_{*} \in \mathscr{T}-$ решение задачи (3.14), то оченка Кукса-Ольмана (3.13) является решением исходной задачи (1.1)-(1.2).

Этот красивьй результат принадлежит В. Б. Меласу [38], который также показал, что двойственность (3.14) остается справедлива с матричным множеством $\mathscr{T}=\mathscr{T}_{1}:=$ conv $\mathscr{T}_{0}$, где $\mathscr{T}_{0}:=\left\{e e^{T} \mid e \in \Theta^{0}\right\}$, а также с множеством $\mathscr{T}_{1}^{\prime}$, полученньм из $\mathscr{T}_{1}$ заменой поляры $\Theta^{0}$ на множество всех ее крайних точек. Это следует из теоремы 1.4.9 и замечания 1.4.10.

Отметим, что двойственная задача (3.14) так же, как и исходная задача (1.2), является задачей минимизации. Поэтому, следуя Auchmuty [69], мы будем назьвать задачи (3.12) и (3.14) аномальными двойственными задачами. 
СлЕДСТВИЕ 3.3.4. Предыдущее следствие остается верныл для матричного множества $\mathscr{T}=\mathscr{T}_{0}$.

Конечно, все результаты этого параграфа справедливы также для хорошо обусловленных задач и когда регрессионная матрица $A$ имеет полный ранг $m$. В этих случаях матрица $A^{T} K^{-1} A+T$ оказьвается невырожденной. Тогда аномальная двойственная задача далее упрощается и очень сильно походит на обыкновенную двойственную задачу (2.1) с априорным множеством $\Theta$, замененным на его поляру $\Theta^{0}$.

СЛЕДСТВИЕ 3.3.5. Пусть множество $\mathscr{K}=\{K\}$ будет одноточечнымм, $\Theta$ будет выпуклым. чентрально-симметричным телом, а регрессионная матрица $А$ имеет полный ранг $m$. Обозначим $F:=\left(A^{T} K^{-1} A\right)^{-1}$. Тогда

$$
D_{\min }=\langle F c, c\rangle-\max _{e \in \Theta^{0}} \frac{\langle F c, e\rangle^{2}}{1+\langle F e, e\rangle} .
$$

Если $e_{*} \in \Theta^{0}$ - решение аномальной двойственной задачи (3.15) и $T_{*}=e_{*} e_{*}^{T}$, то оценка Кукса-Ольмана (3.13) является решением исходной задачи (1.1)-(1.2).

ДокАЗАТЕЛЬСТво. В силу следствия 3.3 .4 имеем

$$
D_{\min }=\min _{e \in \Theta^{0}}\left\langle\left(A^{T} K^{-1} A+e e^{T}\right)^{-1} c, c\right\rangle .
$$

Сочетая это с тождеством (1.4.22), получаем (3.15).

По-видимому, последние два результата являются новыми. Заметим, что в отличие от следствия 3.3.3, поляру $\Theta^{0}$ нельзя в них заменить на множество всех ее крайних точек, и что размерность задачи (3.15) гораздо меньше, чем размерность задачи (3.14) (минимум в (3.15) берется на векторном множестве, тогда как в (3.14) он берется на матричном множестве).

\section{§.4. Несимметричные ограничения на параметры регрессии}

Если априорное множество $\Theta$ не симметрично относительно нуля, все результаты, представленные в этой главе, по-прежнему применимы, но множество $\Theta$ следует симметризовать относительно нуля.

ТЕОРемА 3.4.1. Пусть $\Theta$ будет произвольным выпуклым мелом, а

$$
\Omega:=\left\{\theta=\left(\theta_{1}-\theta_{2}\right) / 2: \theta_{1}, \theta_{2} \in \Theta\right\}
$$

будет его симметричным дубликатом. Тогда

$$
D_{\min }=\max _{K \in \mathscr{K}, \theta \in \Omega} \frac{\langle c, \theta\rangle^{2}}{1+\left\langle K^{-1} A \theta, A \theta\right\rangle} .
$$

Если $K_{*} \in \mathscr{K}, \theta_{*} \in \Omega-$ - решение двойственной задачи (4.2), а $\hat{l}_{*}=\left\langle x_{*}, \eta\right\rangle-$ это оценка (2.2), то оценка

$$
\tilde{l}_{*}=\hat{l}_{*}-\left[\delta^{*}\left(A^{T} x_{*}-c \mid \Theta\right)-\delta^{*}\left(c-A^{T} x_{*} \mid \Theta\right)\right] / 2
$$

является решением исходной задачи (1.1)-(1.2). 
ДокАЗАТЕЛЬСтво. Для фиксированных $x \in \mathbb{R}^{N}$ и $t \in \mathbb{R}$ максимум функции $\left(\left\langle A^{T} x-c, \theta\right\rangle+t\right)^{2}$ на множестве $\Theta$ сводится к максимуму функции $(\alpha+t)^{2}$ на отрезке $a \leqslant \alpha \leqslant b$, где

$$
\begin{gathered}
a=\min _{\theta \in \Theta}\left\langle A^{T} x-c, \theta\right\rangle=-\delta^{*}\left(c-A^{T} x \mid \Theta\right), \\
b=\max _{\theta \in \Theta}\left\langle A^{T} x-c, \theta\right\rangle=\delta^{*}\left(A^{T} x-c \mid \Theta\right) .
\end{gathered}
$$

Ясно, что максимум функции $(\alpha+t)^{2}$ достигается на границе отрезка $[a, b]$. Далее, для фиксированного $x \in \mathbb{R}^{N}$ мы должны найти $\min _{t \in \mathbb{R}} \max \left[(a+t)^{2},(b+t)^{2}\right]$. Очевидно, этот минимум достигается при

$$
t=-(a+b) / 2
$$

и равен $[(b-a) / 2]^{2}=\delta^{* 2}\left(A^{T} x-c \mid \Omega\right)$. Таким образом, исходная задача (1.1)-(1.2) принимает вид

$$
D_{\min } / 2=\min _{x \in \mathbb{R}^{N}}\left\{D(x)+\delta^{* 2}\left(A^{T} x-c \mid \Omega\right) / 2\right\}
$$

причем на этот раз $\delta^{*}(z \mid \Omega)=\left[\delta^{*}(z \mid \Theta)+\delta^{*}(-z \mid \Theta)\right] / 2$ является опорной функцией симметричного множества (4.1).

Равенство (4.2) теперь вытекает непосредственно из теоремы 3.2.1. Если $K_{*} \in \mathscr{K}$, $\theta_{*} \in \Omega$ - это решение двойственной задачи $(4.2)$, а $\hat{l}_{*}=\left\langle x_{*}, \eta\right\rangle$ - это оценка (2.2), то оцениватель $x_{*} \in \mathbb{R}^{N}$ дает решение задачи (4.6), так что согласно (4.4) и (4.5) минимаксная оценка $\tilde{l}_{*}=\left\langle x_{*}, \eta\right\rangle+t_{*}$ принимает вид (4.3).

СлЕДСТвИЕ 3.4.2. Если $\Theta=\theta_{0}+\Theta^{\prime}$, әде $\Theta^{\prime}$ - выпуклое иентрально-симмет-

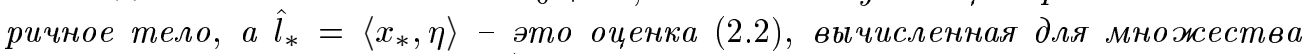
$\Theta^{\prime}$, то $\Omega=\Theta^{\prime}$ и оченка $\hat{l}_{*}=\hat{l}_{*}-\left\langle A^{T} x_{*}-c, \theta_{0}\right\rangle$ является решением исходной задачи (1.1)-(1.2).

ЗАКлюченИЕ. В настоящее время существует несколько путей использования гладкости в сопряженном пространстве. Исторически двойственная связь гладкости со строгой (или равномерной) вьпуклостью первоначально была обнаружена в функциональном анализе (см. [62], [91], [93], [98]). Из изложенного вьше видно, что из гладкости автоматически следует вьпуклость. Такой подход (в сочетании с предельными переходами) весьма плодотворен, и весьма заманчиво было бы его применить для осмысления с общих позиций вьпуклого анализа многих разрозненных результатов, по существу относяшихся к вьпуклому анализу, которые иньми методами были получены в автоматическом управлении [59], [100], спектральной оптимизации [1] и линейной алгебре (в теории числовых образов).

Автор благодарит С. М. Асеева и В. М. Тихомирова за помощь в этой работе. Данная работа вьполнена при частичной поддержке РФФИ. 


\section{СПИСОК ЛИТЕРАТУРЫ}

[1] Абрамов Ю.Ш. Вариационные методы в теории операторных пучков. Спектральная оптимизация. Л.: Изд-во ЛГУ, 1983.

[2] Алексеев В. М., Тихомиров В.М., Фомин С. В. Оптимальное управление. М.: Наука, 1979.

[3] Ананьев Б.И. Минимаксная линейная фильтрация многошаговых процессов с неопределенными распределениями возмущений // Автоматика и телемеханика. 1993. № 10. C. $131-139$.

[4] Бадриев И.П., Карчевский М. М. Методы двойственности в прикладных задачах. Изд-во Казанского ун-та, 1987.

[5] Бажинов И. К., Почукаев В. Н. Оптимальное планирование навигационных измерений в космическом полете. М.: Машиностроение, 1976.

[6] Бахвалов Н. С. Об оптимальности линейных методов приближения операторов на выпуклых классах функций // Журн. вычисл. матем. и матем. физики. 1971. Т. 11. № 4. C. 1014 .

[7] Бахшиян Б. Ц., Назиров Р. Р., Эльясберг П. Е. Определение и коррекция движения. Гарантирующий подход. М.: Наука, 1980.

[8] Бахшиян Б. Ц., Соловьёв В.Н. Применение теоремы двойственности к задаче оптимального гарантированного оценивания // Космич. исслед. 1990. Т. 28. № 2. С. 163-169.

[9] Беллман Р. Введение в теорию матриц. М.: Мир, 1969.

[10] Бураго Ю. Д., Залгаллер В.А. Достаточные признаки выпуклости // Записки научн. семинаров ЛОМИ. 1974. Т. 45. С. 3-52.

[11] Голубев Г. А. Минимаксная линейная фильтрация динамических процессов с дискретньм временем // Автоматика и телемеханика. 1984. № 2. С. 72-81.

[12] Гусев М.И. Оптимальность линейных алгоритмов в задачах гарантированного оценивания // Техническая кибернетика. 1994. №3. С. 87-95.

[13] Гусев М.И. О структуре оптимальных минимаксных оценок в задачах гарантированного оценивания // Докл. АН. 1992. Т. 322. № 5. С. 832-835.

[14] Де Гроот М. Оптимальные статистические решения. М.: Мир, 1974.

[15] Ефимов Н. В., Стечкин С. Б. Опорные свойства множеств в банаховых пространствах и чебышёвские множества // Докл. АН СССР. 1959. Т. 127.№ 2. С. 254-257.

[16] Закс Ш. Теория статистических выводов. М.: Мир, 1975.

[17] Ибрагимов И.А., Хасьминский Р. З. О непараметрическом оценивании значения линейного функционала в гауссовском белом шуме // Теория вероятн. и ее примен. 1984. T. 29. № 1. C. 19-32.

[18] Ибрагимов И.А., Хасьминский Р.З. Об оценивании линейных функционалов в гауссовском шуме // Теория вероятн. и ее примен. 1987. Т. 32. №1. С. 35-44.

[19] Иоффе А. Д., Тихомиров В. М. Теория экстремальных задач. М.: Наука, 1974.

[20] Кац И.Я., Куржанский А. Б. Минимаксная многошаговая фильтрация в статистически неопределенных ситуациях // Автоматика и телемеханика. 1978. № 11. С. 79-88.

[21] Колмогоров А.Н., Фомин С. В. Элементы теории функций и функционального анализа. М.: Наука, 1976.

[22] Красовский Н. Н. К теории управляемости и наблюдаемости линейных динамических систем // Прикл. матем. и мех. 1964. Т. 28. № 1. С. 3-14.

[23] Кукс А. Минимаксная оценка коэффициентов регрессии // Изв. АН Эст. ССР. Сер. физ.-мат. 1972. Т. 21. № 1. С. 73-78.

[24] Кукс А., Ольман В. Минимаксная линейная оценка коэфффициентов регрессии // Изв. АН Эст. ССР. Сер. физ.-мат. 1972. Т. 21. №1. С. 66-72.

[25] Кукс А., Ольман В. Минимаксная линейная оценка коэффициентов регрессии // Изв. АН Эст. ССР. Сер. физ.-матем. 1971. Т. 20. № 1. С. 480-482.

[26] Куржанский А. Б. Управление и наблюдения в условиях неопределенности. М.: Наука, 1977. 
[27] Куржанский А. Б. Задача идентификации: теория гарантирующих оценок (обзор) // Автоматика и телемеханика. 1991. № 4. С. 3-26.

[28] Куркин О.М., Коробочкин Ю. Б., Шаталов С. А. Минимаксная обработка информации. М.: Энергоатомиздат, 1990.

[29] Левчук О.В., Федотов А. М. Построение минимаксных решений линейных некорректных задач со случайными ошибками в данных // Журн. вычисл. матем. и матем. физики. 1988. Т. 28. №6. С. $825-834$.

[30] Легостаева И.Л., Ширяев А.Н. Минимаксные веса в задаче выделения тренда случайного процесса // Теория вероятн. и ее примен. 1971. Т. 16. № 2. С. 339-345.

[31] Леман Э. Теория точечного оценивания. М.: Наука, 1991.

[32] Лидов М.Л., Бахшиян Б.Ц., Матасов А. И. Об одном направлении в проблеме гарантирующего оценивания (обзор) // Космич. исслед. 1991. Т. 29. № 5. С. 659-684.

[33] Липцер Р.Ш., Ширяев А.Н. Статистика случайных процессов. М.: Наука, 1974.

[34] Магарил-Ильяев Г. Г., Осипенко К. Ю. Об оптимальном восстановлении функционалов по неточным данным // Матем. заметки. 1991. Т. 50. № 6. С. 85-93.

[35] Марчук А.Г., Осипенко К. Ю. Наилучшее приближение функций, заданных с погрешностью в конечном числе точек // Матем. заметки. 1975. Т. 17. № 3. С. 359.

[36] Матасов А.И. Об оптимальности линейных алгоритмов гарантированного оценивания, части I, II // Космич. исслед. 1988. Т. 26. № 5. С. $643-653 ;$ 1988. Т. 26. № 6 . C. $803-812$.

[37] Матасов А.И. Оптимальность линейных алгоритмов в задаче о "наихудшей корреляции" // Вестн. МГУ. Сер. 1. Матем., мех. 1989. №1. С. 61-64.

[38] Мелас В.Б. О выборе плана эксперимента и метода оценивания при наличии априорных сведений о параметрах // Математические методы планирования эксперимента. Новосибирск: Наука, 1981. С. 155-173.

[39] Обен Ж. П., Экланд И. Прикладной нелинейных анализ. М.: Мир, 1988.

[40] Пинелис И.Ф. О минимаксном риске // Теория вероятн. и ее примен. 1990. Т. 35. № 1. C. $92-97$.

[41] Пинелис И.Ф. О минимаксном оценивании регрессии // Теория вероятн. и ее примен. 1990. Т. 35. № 3. С. 494-505.

[42] Поляк Б. Т. Введение в оптимизацию. М.: Наука, 1983.

[43] Пшеничный Б. Н. Необходимые условия экстремума. 2-е изд. М.: Наука, 1982.

[44] Пшеничный Б. Н., Покотило В. Г. Минимаксный подход к оценке параметров линейной регрессии // Изв. АН ССР. Сер. Техн. кибернетика. 1983. № 2. С. 94-102.

[45] Рокафеллар Р. Т. Выпуклый анализ. М.: Наука, 1973.

[46] Соловьёв В.Н.Двойственность некоторых невыпуклых экстремальных задач // Журн. вычисл. матем. и матем. физики. 1987. Т. 27. № 2. С. 459-463.

[47] Соловьёв В.Н.Двойственность невыпуклых экстремальных задач // Докл. ды АН CCCP. 1990. T. 314. № 1. C. 135-138.

[48] Соловьёв В.Н. Некоторые алгоритмы квадратичного программирования и оптимального гарантирующего оценивания // Автоматика и телемеханика. 1990. № 9. С. 67-73.

[49] Соловьёв В.Н. Одна теорема о минимаксе // Тезисы доклада Всесоюзной конференции "Негладкий анализ и его приложения к математической экономике". Баку: Изд. Элм, 1991.

[50] Соловьёв В.Н. Двойственные алгоритмы оптималњного гарантирующего оценивания // Космич. исслед. 1992. Т. 30. №1. С. 10-24.

[51] Соловьёв В.Н. О линейности оптималшных алгоритмов гарантирующего оценивания // Космич. исслед. 1994. Т. 32. № 2. С. 122-124.

[52] Соловьёв В.Н. Двойственные алгоритмы оптималшного гарантирующего оценивания и усеченный метод наименьших квадратов // Космич. исслед. 1995. Т. 33. № 1. С. 3-11.

[53] Соловьёв В. Н. Минимаксно-байесовское оценивание на классах распределений с ограниченными вторыми моментами // УМН. 1995. Т. 50. № 4. С. 171-172.

[54] Соловьёв В.Н. Минимаксное и минимаксно-байесовское оценивание параметров линейной регрессии // УМН. 1996. Т. 51. № 3. С. 211-212. 
[55] Соловьёв В.Н.О Связи выпуклости и гладкости // Тезисы докладов III Международной конференции женщин-математиков (29 мая - 2 июня 1995 г.). Воронеж: ВИПКРО, 1995. C. 60 .

[56] Соловьёв В.Н. О связи выпуклости и гладкости. II // Тезисы докладов Воронежской весенней математической школы "Современные методы в теории краевых задач", "Понтрягинские чтения-VII" (17- 23 апреля 1996). Воронеж: ВГУ. С. 168.

[57] Федотов А. М., Левчук О.В. Оптимальные линейные оценки линейных функционалов от решений операторных уравнений // Докл. АН СССР. 1986. Т. 287. № 1. С. 63-66.

[58] Федотов А. М. Некорректные задачи со случайньми ошибками в данных. Новосибирск: Наука, 1990.

[59] Фрадков А. Л. Теоремы двойственности в некоторых невыпуклых экстремальных задачах // Сиб. матем. журн. 1973. Т. 14. № 2. С. 357-383.

[60] Хьюбер П. Робастность в статистике. М.: Мир, 1984.

[61] Черноусько Ф. Л. Оценивание фазового состояния динамических систем. М.: Наука, 1988.

[62] Шмульян В. Л. Sur la structure de la sphere unitare dans l'espace de Banach // Матем. сб. 1941. Т. 9. С. $545-561$.

[63] Шор Н.3. Об одном подходе к получению глобальных экстремумов в полиномиальных задачах математического программирования // Кибернетика. 1987. № 5. С. 102-106.

[64] Шор Н. З., Стеценко С. И. Квадратичные экстремальные задачи и недифференцируемая оптимизация. Киев: Наукова Думка, 1989.

[65] Экланд И., Темам Р. Выпукльй анализ и вариационные проблемы. М.: Мир, 1979.

[66] Asplund E. Frechet-Differentiability of Convex Functions // Acta Math. 1968. V. 121. P. 31-47.

[67] Asplund E., Rockafellar R. T. Gradients of Convex Functions // Trans. Amer. Math. Soc. 1969. V. 139. P. 443-467.

[68] Asplund E. Chebyshev Sets in Hilbert Space // Trans. Amer. Math. Soc. 1969. V. 144. P. $235-240$.

[69] Auchmuty G. Duality for Nonconvex Variational Principles // J. Differential Equations. 1983. V. 50. P. $80-145$

[70] Basar T., Mintz M. On a Minimax Estimate for the Mean of a Normal Random Vector under a Generalized Quadratic Loss Function // Ann. Statist. 1973. V. 1. № 1.

[71] Berger J.O. Statistical Decision Theory and Bayesion Analysis, 2nd ed. New York: Springer, 1985

[72] Bickel P. J. Minimax Estimation of the Mean of a Normal Distribution when the Parameter Space is Restricted // Ann. Statist. 1981. V. 9. P. 1301-1309.

[73] Brondsted A., Rockafellar R. T. On the Subdifferentiability of Convex Functions // Proc. Amer. Math. Soc. 1965. V. 16. P. 605-611.

[74] Bunke O. Least Squares Estimators as Robust and Minimax Estimators // Math. Operationsforsch. und Statist. 1975. V. 6. № 5. P. 687-688.

[75] Casella G., Strawderman W. E. Estimating a Bounded Normal Mean // Ann. Statist. 1981. V. 9. P. 870-878.

[76] Craven B.D., Gwinner J., Jeyakumar V. Noncovex Theorems of the Alternative and Minimization // Optimization. 1987. V. 18. № 2. P. 151-163.

[77] Ekeland I. Legendre Duality in Nonconvex Optimization and Calculus of Variations // SIAM J. Control and Optimization. 1977. V. 15. P. 905-934.

[78] Martin C. J., Mintz M. Robust Filtering and Prediction for Linear Systems with Uncertain Dynamics: A Game Theoretic Approach // IEEE Trans. on Autom. Control. 1983. V. AC-28. P. 888-896.

[79] Milanese M., Tempo R. Optimal Algorithms Theory for Robust Estimation and Prediction // IEEE Trans. on Autom. Control. 1985. V. AC-80. № 8. P. 730-738.

[80] Milanese M., Vicino A. Optimal Estimation Theory of Dynamic Systems with Set Membership Uncertainty // Automatica. 1991. V. 27. №6. P. 997-1009. 
[81] Mintz M. A Kalman Filter as a Minimax Estimator // J. Optimization Theory Appl. 1972. V. 9. № 2 .

[82] Neumann J. fon. Zur Theorie der Gesellschaftspiele // Math. Ann. 1928. V. 100. P. 295-320.

[83] Packel E. W. Do linear problems have linear optimal algorithms? // SIAM Rev. 1988. V. 30. № 3. P. 388-403.

[84] Poor H. V., Verdu S. Minimax Linear Observers and Regulators for Stochastic Systems with Uncertain Second Order Statistics // IEEE Trans. Automat. Control. 1984. V. AC-29. P. 499-511.

[85] Pukelsheim F., Studeen W. J. E-optimal designs for polynomial regression // Ann. Statist. 1993. V. 21. № 1. P. 402-415.

[86] Sacks J., Ylvisaker D. Linear Estimation for Approximately Linear Models // Ann. Statist. 1978. V. 6. P. 1122-1137.

[87] Soloviov V. Duality for Nonconvex Optimization and its Applications // Anal. Math. 1993. V. 19. № 4. P. 297-315.

[88] Soloviov V. Minimax Estimation and the Least Squares Method // Stochastics and Stochastics Reports. 1993. V. 42. P. 209-223.

[89] Vandelinde V.D. Robust Properties of Solutions to Linearquadratic Estimation and Control Problems // IEEE Trans. Automat. Control. 1977. V. AC-22. № 1. P. 138-139.

[90] Wald A. Statistical Decision Functions. New York: Wiley, 1950.

[91] Deville R., Godefroy G., Zizler V. Smoothness and Renormings in Banach Spaces. New York: Wiley, 1993.

[92] Giles J.R. Convex Analysis with Application in Differentiation of Convex Functions // Res. Notes Math. № 58. Pitman, 1982.

[93] Phelps R. R. Convex Functions, Monotone Operators and Differentiability // Lecture Notes in Math. 1993 (2nd ed.). V. 1364.

[94] Балаганский В. С. Дифференцируемость по Фреше функции расстояния и структура множества // Матем. заметки. 1988. Т. 44. №6. С. 725.

[95] Власов Л.П. Несколько теорем о чебьшёвских множествах // Матем. заметки. 1972. T. 11. № 2. С. 135-144.

[96] Гольштейн Е. Г. Теория двойственности в математическом программировании и ее приложения. М.: Наука, 1971.

[97] Данфорд Н., Шварц Дж. Линейные операторы. Общая теория. М.: ИЛ, 1962.

[98] Дистель Дж. Геометрия банаховых пространств. Киев: Вища школа, 1980.

[99] Смоляк С.А.Об оптимальном восстановлении функций и функционалов от них // Дисс. ... канд. физ.-матем. наук. М.: МГУ, 1965.

[100] Якубович В.А., Фрадков В.Л. $S$-процедура и соотношение двойственности в невыпуклых задачах квадратичного программирования // Вестник ЛГУ. Сер. матем., мех. 1973. T. 1. № 1. C. 81-87.

[101] Montejano L., Schcepin E. V. A characterization of convex sets in term of acyclic support sets // Bull. London Math. Soc. 1996. V. 28. № 5. P. 501-504. 